ISSN 0373-580 X

Bol. Soc. Argent. Bot. 49 (4): 559-580. 2014

\title{
VEgETACIÓN dE AFLORAMIENTOS CARBONÁTICOS DE MONTAÑAS DEL CENTRO DE ARgentina
}

\author{
JUAN JOSÉ CANTERO1,4,6*, JORGE A. SFRAGULLA2,3, CÉSAR NÚÑEZ1', JOSÉ MULKO1, \\ ALDO A. BONALUMI ${ }^{2,3}$, ANDREA AMUCHASTEGUI ${ }^{1}$, GLORIA E. BARBOZA ${ }^{4,5,6}$, FRANCO \\ CHIARINI ${ }^{4,6}$ y LUIS ARIZA ESPINAR ${ }^{4,6}$
}

\begin{abstract}
Resumen: En las montañas del centro de Argentina, con una intensa fragmentación y reemplazos de la vegetación natural, los afloramientos rocosos constituyen hábitats importantes para la conservación de la biodiversidad, como refugios de especies endémicas y raras. En este trabajo se exploró la variación composicional local de la vegetación en afloramientos de mármoles cálcicos y dolomíticos de las sierras de Córdoba y se compararon las asociaciones entre diferentes tipologías geoquímicas y la composición florística en un gradiente altitudinal. Se relevaron cuatro afloramientos adyacentes de tipologías geoquímicas diferentes localizados en diferentes posiciones en un gradiente de altitud desde 1000 hasta 1800 msm, estimándose abundancia-cobertura de todas las plantas vasculares en 216 censos. Los atributos composicionales estructurales de la vegetación revelaron diferencias significativas entre litologías similares en diferentes estratos altitudinales y entre diferentes litologías para un mismo estrato altitudinal. La composición florística local está relacionada con la tipología geoquímica de los afloramientos y la altitud se sobrepone a ese patrón. Se establecieron relaciones entre la presencia de diferentes especies vegetales y tipologías de rocas calcíticas y dolomíticas de manera independiente a su posición en el gradiente de altitud. Se confirma la importancia de estas variables en la estructuración del hábitat y filtrado abiótico de especies y la importancia de su conocimiento para establecer prioridades en su conservación.
\end{abstract}

Palabras clave: Afloramientos carbonáticos, altitud, geoquímica, composición florística.

\begin{abstract}
Summary: Vegetation of carbonate outcrops in mountains of central Argentina. In mountains of Central Argentina, which have severe fragmentation and replacement of natural vegetation, rocky outcrops are important habitats for biodiversity conservation, as refuges for endemic and rare species. In this paper, we explore the local compositional variation of vegetation in calcium and dolomitic outcrops of Sierras de Córdoba and compare associations between different geochemical types and floristic composition along a local altitudinal gradient. Four adjacent outcrops of different geochemical types located in different positions in a local elevation gradient from 1000 to 1800 m.a.s.l. were selected and cover-abundance of all vascular plants at 216 rélevés, was estimated. Significative differences of compositional vegetation attributes occurred between the different lithologies and altitudinal strata. At the local scale, the floristic composition appeared related to the geochemical typology of outcrops and this pattern is overlapped with altitude. It was possible to establish relationships between the presence of different vascular plants and different types of calcitic and dolomitic rocks, independently of their position in the altitudinal gradient. The importance of these variables in structuring the habitat and filtering of species is confirmed, and its importance in looking for priorities in conservation of these singular habitats.
\end{abstract}

Key words: Carbonate outcrops, altitude, geochemistry, floristic composition.

\footnotetext{
${ }^{1}$ Departamento Biología Agrícola, Facultad de Agronomía y Veterinaria, UNRC, Ruta Nac. 36, Km. 601, 5804 Río Cuarto, Córdoba.

${ }^{2}$ Secretaría de Minería, Provincia de Córdoba, Hipólito Yrigoyen 401, 5000 Córdoba.

${ }^{3}$ Facultad de Ciencias Exactas, Físicas y Naturales, UNC, Av. Vélez Sársfield 1611, 5016 Córdoba.

${ }^{4}$ Instituto Multidisciplinario de Biología Vegetal (CONICET-UNC), Córdoba.

${ }^{5}$ Facultad de Ciencias Químicas, UNC. Haya de la Torre y M. Allende s.n., Córdoba.

${ }^{6}$ Museo Botánico Córdoba (UNC).

*Autor para correspondencia: juanjocantero@gmail.com
} 


\section{INTRODUCCIÓN}

En las montañas del centro de Argentina los afloramientos rocosos representan el $90 \%$ de toda la superficie serrana $\left(54.000 \mathrm{~km}^{2}\right)$, de los cuales aproximadamente el $1 \%$ se corresponden a rocas carbonáticas cálcicas y dolomíticas que son el hábitat de una importante endemoflora (Cantero et al., 2011). En el Distrito del Chaco Serrano, la unidad fitogeográfica dominante de estos paisajes geomorfológicos, la fragmentación de la vegetación ha alcanzado valores dramáticos (Zak \& Cabido, 2004) afectando la reproducción y regeneración de varias especies (Aizen \& Feinsinger, 1994a, b; Aguilar \& Galetto, 2004; Chacoff et al., 2004) y promoviendo extinciones locales de muchas de ellas (Cagnolo et al., 2006). Este Distrito ha sido recientemente estudiado desde el punto de vista florístico, entre los 400 y $1700 \mathrm{~m}$, y se han mencionado un total de 106 familias, 476 géneros y 896 especies de plantas vasculares que crecen en el mismo (Giorgis et al., 2011). Se ha sugerido que el manejo dirigido a la conservación del Chaco Serrano debe dar prioridad no solo a los remanentes extensos de vegetación aún existentes en esta ecorregión, sino también a preservar la red regional de interconexiones para favorecer a la viabilidad de poblaciones de las especies raras localizadas en fragmentos singulares del paisaje serrano (Cagnolo et al., 2006). En este sentido, los afloramientos rocosos son hábitats singulares que tienen un papel muy importante como refugios de especies raras, ya sea por los atributos de sus poblaciones o porque están sometidos a perturbaciones frecuentes.

Las yacencias de rocas carbonáticas más importantes en las Sierras de Córdoba, en cuanto a volumen, están constituidas por afloramientos de mármoles cálcicos y dolomíticos, originados por metamorfismo regional de rocas carbonáticas de origen químico, que afloran asociados a otras rocas del basamento metamórfico, especialmente gneises, anatexitas, anfibolitas y en algunos casos a serpentinitas (Sfragulla et al., 1999). Las rocas carbonáticas se cuentan entre las sustancias minerales con mayor cantidad y diversidad de usos, siendo empleadas tanto en forma natural (bloques de mármol para uso ornamental), como carbonato o dolomita (industria de la pintura, papel, vidrio, química y cerámica los más puros y para fabricación de revestimientos y mosaicos los que tienen más impurezas), o como óxidos de calcio y magnesio obtenidos a partir de la calcinación. Con todos esos fines, estas rocas tienen una alta demanda industrial, ocupando el tercer lugar en tonelaje de producción en la provincia de Córdoba con unos 3 millones de toneladas para 2009 (Sfragulla com.per.) y convierten a sus afloramientos en sitios altamente vulnerables desde el punto de vista de conservación de su flora. La función y el valor de estos afloramientos carbonáticos en relación a la conservación de la biodiversidad podrían ser considerados de dos maneras: (1) proveer de hábitats para especies sobre-utilizadas, como las medicinales, forrajeras y las usadas como combustibles, contribuyendo a mantener y aumentar la diversidad regional y (2) ser fuentes de especies para la recolonización de sitios degradados circundantes.

En diferentes ecorregiones del mundo se ha reconocido que los afloramientos rocosos constituyen hábitats particularmente importantes desde el punto de vista de conservación de la biodiversidad por la riqueza de sus endemismos, la presencia de especies raras y su valor como refugio de especies amenazadas (Wardle, 1971, 1991; Rogers \& Walter, 2002; Burke et al., 2003; Hunter, 2003; de Lange et al., 2004; Wiser \& Buxton, 2009; Esgario et al., 2009; Sadler \& Bradfield, 2010). El conocimiento de las especies que allí crecen seguidos de estudios sobre su autoecología son prerrequisitos para poder generar acciones serias de conservación (Smith \& Cleef, 1988; Barthlott et al., 1993; Alves \& Kolbek, 1994; Porembski et al., 1994; Giuletti et al., 1997; Michelangeli, 2000).

Los factores determinantes de la composición florística de las comunidades rupícolas varían de acuerdo con la escala considerada, regional y local (Harrisson et al., 2006), y en la mayoría de las situaciones representan islas de comunidades xéricas dentro de una matriz de vegetación mesofítica (Jacobi et al., 2007). Los límites geográficos de las comunidades están condicionados por el clima que define unidades regionales de vegetación (Ortiz \& Rodríguez Oubiña, 1993). En la escala local, los factores activos en los hábitats rocosos se relacionan con: 1) características de la roca donde crece la vegetación, tal como las propiedades y composición química, 2) patrones de erosión y fracturación -que a su vez están relacionadas con la composición- (rocas calcáreas, cuarcitas, 


\section{J. J. Cantero et al. - Vegetación de afloramientos carbonáticos del centro de Argentina}

granitos, etc.) y condiciones ambientales locales, 3) variaciones topográficas y microclimáticas, y 4) contexto florístico regional, dinámica sucesional, perturbaciones antrópicas como fuego y ganadería, paleoclima e historia (Porembski et al., 1994; Escudero, 1996; Larson et al., 2000; Seine et al., 2000; Burke, 2002a, b; Wiser \& Buxton, 2008, 2009).

La altitud es un factor discriminante importante de la composición de la vegetación (Ashton \& Webb, 1977; Cabido et al., 1990; Maycock \& Fahselt, 1992; Wiser et al., 1996; Wiser \& Buxton, 2009) que incorpora los gradientes directos de precipitación (que se incrementa con la altitud) y temperatura (que decrece con la altitud). En serpentinitas de California, Grace et al. (2007) pudieron establecer asociaciones estrechas entre la variación de la vegetación y las precipitaciones en la escala regional y hallaron patrones más complejos con la relación $\mathrm{Mg} / \mathrm{Ca}$ para las escalas locales. Otros estudios realizados en escalas locales han hallado una estrecha correspondencia entre la composición de las rocas y la variación florística (Mota et al., 2008; Pope et al., 2010).

Se han realizado numerosos estudios florísticos en distintos tipos de afloramientos, en especial en dolomías (Medina Cazorla et al., 2005; Mota et al., 2008); calizas (Fernández Areces et al., 1983; Morat et al., 2001); granitos (Burbanck \& Platt, 1964; Wiser et al., 1996) y serpentinitas (Safford et al., 2005; Grace et al., 2007). La ecología y fitosociología de la vegetación rupícola ha recibido escasa atención en Sudamérica (Gutte, 1986; Martínez Carretero, 1994; Funes \& Cabido, 1995; Galán de Mera et al., 2003; Scarano, 2007; Méndez, 2010). Los afloramientos carbonáticos del centro de Argentina han sido estudiados por Cantero et al. (2011) en una amplia escala regional $(33.600$ $\mathrm{km}^{2}$ ). En estos hábitats las variaciones florísticas encontradas entre los afloramientos no se pudieron atribuir en forma exclusiva a las características químicas del sustrato rocoso ya que la variación ambiental a nivel regional, variable a lo largo del gradiente geográfico (y climático), enmascara la variación local ambiental (geoquímica, altitud) entre los afloramientos (Cantero et al., 2011).

Los objetivos de este trabajo son explorar la variación en la composición local de la vegetación en afloramientos carbonáticos cálcicos y dolomíticos del Distrito Chaqueño Serrano centro-argentino y comparar las asociaciones entre diferentes tipologías geoquímicas y la composición florística en un gradiente altitudinal. Con el objetivo de entender mejor la vegetación de estos afloramientos nos preguntamos: 1) ¿la composición florística a nivel local es uniforme en estos afloramientos o varía?, 2) ¿el gradiente altitudinal a nivel local se sobrepone a la variación geoquímica entre afloramientos?

\section{Materiales y MÉtodos}

\section{Área de Estudio}

En las Sierras de Córdoba (Argentina) existen tres grandes lineamientos regionales con orientación submeridional (D’Aloia, 1959), Cordón Oriental, Cordón Central y Cordón Occidental en donde se localizan los afloramientos carbonáticos (Cantero et al., 2011). A los fines de explorar una escala local, se seleccionó dentro de esa variabilidad regional, un área homogénea desde el punto de vista fitogeográfico, es decir que comparte una misma historia florística y pool regional de especies (Cantero et al., 2011), con afloramientos de geoquímicas contrastantes y cercanos entre sí (Fig. 1). En los afloramientos rocosos en general $\mathrm{y}$ en los pocos conocidos desde el punto de vista botánico como los de este estudio, las unidades florístico-ecológicas concretas reconocibles a campo son difíciles de detectar (Heywood, 1953). Por ello y en función del objetivo de este trabajo, se seleccionaron afloramientos planares, mayores de una hectárea y sin ningún signo de laboreo minero (Sadler \& Bradfield, 2010). De esa manera quedaron seleccionados cuatro afloramientos: Los Cienegueros, Cuchi Yaco, Piedra Sonadora, todos en el Cordón Central y Mesa La Argentina en el Cordón Occidental. Los cuatro afloramientos cercanos seleccionados representaron réplicas de dos tipologías geoquímicas diferentes dentro de un área de $412 \mathrm{~km}^{2}$ aproximadamente. Los cuatro afloramientos cercanos fueron representativos de dos tipologías geoquímicas y dos altitudes diferentes (ver detalles en Tabla 1).

Las precipitaciones varían entre los 700 y 800 mm (Bianchi \& Cravero, 2010). La temperatura media anual varía entre 13 y $17^{\circ} \mathrm{C}$ y las temperaturas medias del mes de enero oscilan entre los 18 y $30^{\circ} \mathrm{C}$ y la de julio entre los 4 y $18^{\circ} \mathrm{C}$ (INTA, 2014). Las 
Bol. Soc. Argent. Bot. 49 (4) 2014

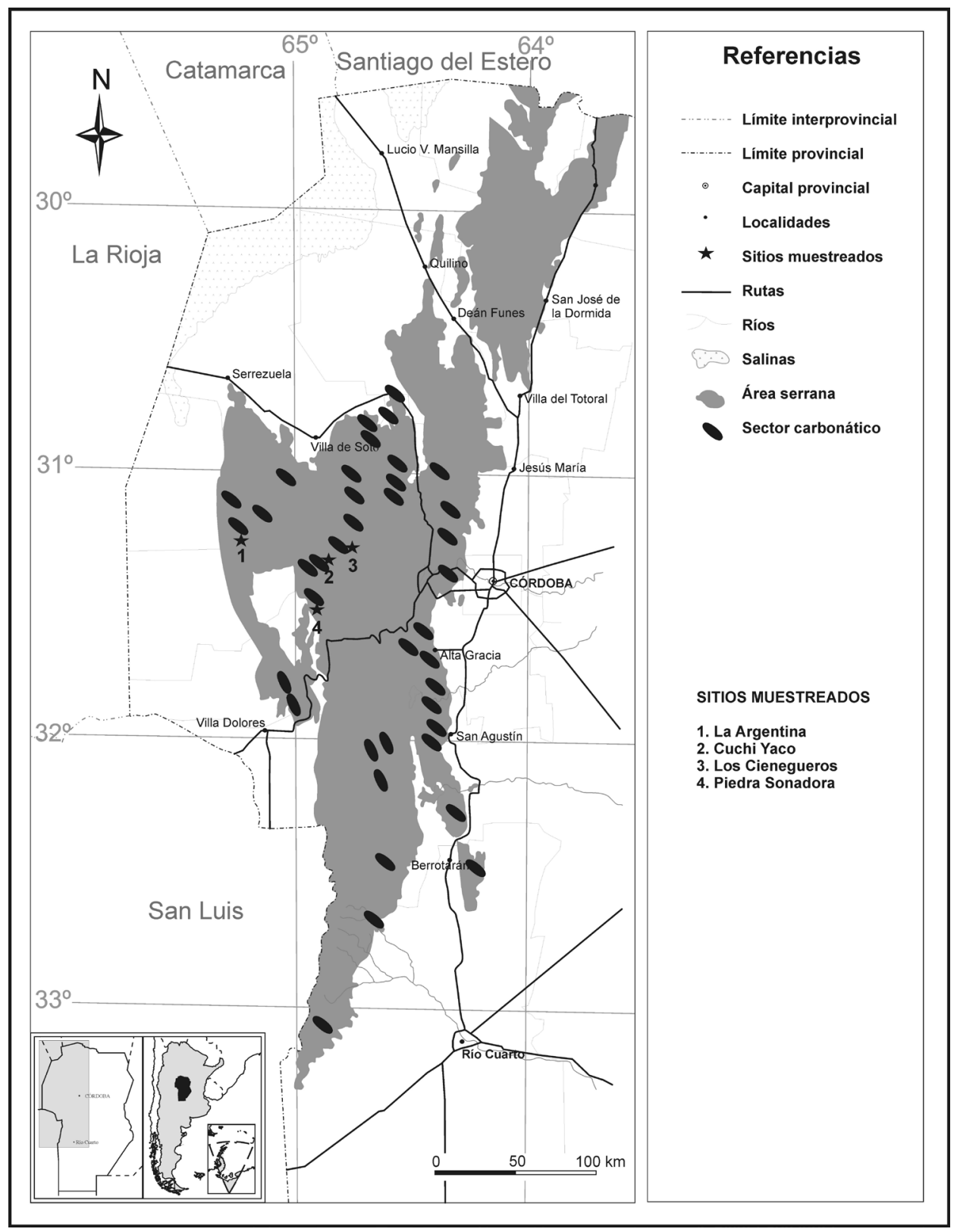

Fig. 1. Área de estudio con el detalle de la localización de los afloramientos carbonáticos estudiados.

Tabla 1. Localización geográfica de los sitios de estudio.

\begin{tabular}{|c|c|c|c|c|c|c|}
\hline Afloramiento & \multicolumn{2}{|c|}{ Localización geográfica } & Altitud & $\begin{array}{l}\text { Área } \\
\left(\mathrm{m}^{2}\right)\end{array}$ & Tipología geoquímica & Simbología \\
\hline La Argentina & S $31^{\circ} 13^{\prime} 15,3$ & W $65^{\circ} 19^{\prime} 13,82^{\prime \prime}$ & 1100 m.s.m. & 91.719 & Calcítica & $\mathrm{Cb}$ \\
\hline Cuchi Yaco & S $31^{\circ} 19^{\prime} 41,3$ & W 65 02' 20,6"' & 1000 m.s.m. & 50.775 & Dolomítica & $D b$ \\
\hline Piedra Sonadora & $S 31^{\circ} 29^{\prime} 9,4$ & W 64 $58^{\prime} 48,9^{\prime \prime}$ & 1600 m.s.m. & 56.001 & Calcítica & $\mathrm{Ca}$ \\
\hline Los Cienegueros & $S 31^{\circ} 17^{\prime} 38,5$ & W 64 $49^{\prime} 34,5^{\prime \prime}$ & 1800 m.s.m. & 113.558 & Dolomítica & $D a$ \\
\hline
\end{tabular}




\section{J. J. Cantero et al. - Vegetación de afloramientos carbonáticos del centro de Argentina}

temperaturas absolutas oscilan entre los $-8,5$ a $42^{\circ} \mathrm{C}$ (SMN, 2014). La vegetación de los afloramientos pertenece a la unidad fitogeográfica del Chaco Serrano (Cabrera, 1976) y el tipo dominante de vegetación en todos ellos es un arbustal bajo con gramíneas en su matriz.

\section{Muestreo}

Para caracterizar el quimismo de los afloramientos, se empleó la clasificación propuesta por Sfragulla et al. (1999) quienes denominan como calcáreos con tendencia calcítica a aquellos afloramientos que tienen entre $30 \%-52 \%$ de $\mathrm{CaO}$ y entre $0 \%$ y $11 \%$ de $\mathrm{MgO}$, y calcáreos con tendencia dolomítica los que poseen entre $30 \%-35 \%$ de $\mathrm{CaO}$ y entre $13 \%-21 \%$ de $\mathrm{MgO}$. El trabajo de colección se extendió desde octubre de 2009 a marzo de 2010 y fue llevado a cabo por un equipo interdisciplinario de geólogos, botánicos y ecólogos. La vegetación fue muestreada siguiendo un esquema similar al de Pope et al. (2010) que ha resultado adecuado para este tipo de afloramientos rocosos, y de acuerdo a método desarrollado por Stohlgren et al. (2006). Para ello, se seleccionaron dos puntos en cada uno de los afloramientos seleccionados desde los cuales se extendieron 3 transectas de $90 \mathrm{~m}$ de longitud dirigidas en otras tantas direcciones a $120^{\circ}$ entre sí. En cada una de esas líneas se localizó un punto en forma aleatoria (sitio) donde se situó una unidad de muestreo (círculo) con tres líneas internas $(\mathrm{T} 1, \mathrm{~T} 2, \mathrm{~T} 3)$ a $30^{\circ}, 270^{\circ}$ y $150^{\circ}$ grados respectivamente donde se dispusieron 9 cuadrados (censos) de 1 x $1 \mathrm{~m}$ (Fig. 2).

En el interior de cada cuadrado se registró el porcentaje de cobertura de todas las especies de plantas vasculares presentes, de acuerdo a una escala de ocho clases de cobertura, producto de la modificación de la de Braun-Blanquet (1979) (1= rara, con cobertura muy reducida; $2=<1 \% ; 3=1-2$ $\% ; 4=2-5 \% ; 5=6-25 \% ; 6=26-50 \% ; 7=51-75$ $\% ; 8=>75 \%$ ). Se relevó un total de 216 censos distribuidos de manera equitativa (54) en cada uno de los afloramientos seleccionados (Dolomítico alto, Dolomítico bajo, Calcítico alto, y Calcítico bajo). Para corroborar la actualización de los nombres y las abreviaturas de los autores de las especies se usó el Catálogo de las Plantas Vasculares del Cono Sur en su versión online (www.darwin.edu.ar). Todos los ejemplares coleccionados fueron depositados en los herbarios del Museo Botánico de Córdoba

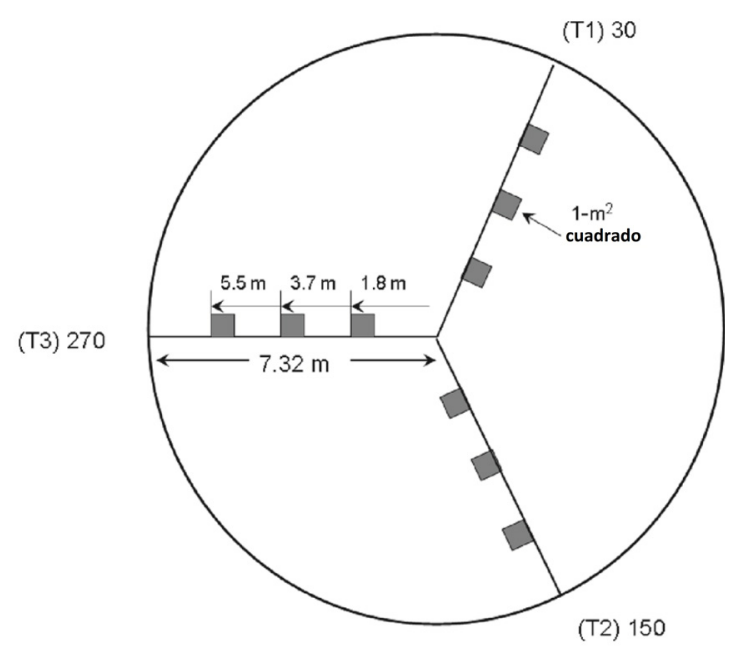

Fig. 2. Unidad de muestreo de la vegetación utilizada en este trabajo y adaptada de Stohlgren et al. (2005) y Pope et al. (2010).

(CORD) y de la UNRC (RIOC). Se consideró como especie endémica local a la que crece solamente en la provincia de Córdoba, endémica regional a la que crece en la provincia de Córdoba y sus provincias limítrofes y finalmente endémica nacional la que tiene una amplia distribución en Argentina. Para las especies identificadas se consideró sus formas de vida según Giorgis et al. (2011). Para establecer la distribución regional de los endemismos se emplearon los trabajos de Cabido et al. (1998), Zuloaga et al. (1994), Zuloaga \& Morrone (1999a, b) y Zuloaga et al. (2008). Para valorar la importancia para la conservación de estos sitios se identificaron las especies medicinales de cada afloramiento mediante las bases de datos de Barboza et al. (2006, 2009).

En cada sitio de relevamiento de la vegetación se realizó también el muestreo litológico para la evaluación posterior de la geoquímica de las rocas. Para ello se constituyó una muestra compuesta, cuidando que la muestra obtenida fuera representativa de la roca aflorante. La totalidad de la muestra original fue triturada, mezclada, sometida a cuarteo (cuarteador Jones) y la fracción de ensayo reducida a polvo (partículas $<$ a 150 micras) mediante molino Siebtechnik con mortero de ágata. Las concentraciones de Calcio (Ca) y Magnesio $(\mathrm{Mg})$ fueron determinadas por espectrofotometría de absorción atómica en un equipo Varian 220 con flamas de Óxido Nitroso-Acetileno y Aire- 
Acetileno en el Laboratorio Geoquímico de la Secretaría de Minería de Córdoba.

\section{Análisis de los datos}

Los atributos composicionales de la vegetación que se calcularon fueron: riqueza, diversidad, equitatividad y dominancia, siguiendo la propuesta de Mc Cune \& Mefford (1999) y para evaluar si hubo diferencias significativas de todos esos atributos para la vegetación de los diferentes afloramientos, se empleo el test de Tuckey, con un análisis previo de los datos para comprobar la normalidad y homogeneidad de varianza.

Las diferencias florísticas entre los dos estratos definidos por la altitud fue evaluada a través de Pruebas de Combinaciones Múltiples (MRPP) y el carácter indicador de los taxones fue explorado con el Análisis de Especies Indicadoras (ISA). Las relaciones entre la vegetación y las variables ambientales fueron evaluadas a través de un Análisis de Redundancia (RDA)(ter Braak, 1995). Todos los cálculos se realizaron usando InfoStat (Di Rienzo et al, 2011), PC-ORD vs. 5 (Mc Cune \& Mefford, 1999) y CANOCO vs.4.5 (ter Braak, 2002).

\section{Resultados}

\section{Características florísticas de los afloramientos}

La flora vascular de los afloramientos carbonáticos relevados está representada por 131 especies distribuidas en 42 familias y 109 géneros. Se hallaron 30 géneros con 32 especies nativas exclusivas de los afloramientos calcíticos y 36 géneros con 37 especies en los afloramientos dolomíticos. La riqueza promedio de especies fue de 14 y la diversidad promedio de $\mathrm{H}=2,5$. Aproximadamente el $54,19 \%$ de las especies pertenecen a cuatro (4) familias. Las familias mejor representadas son: Asteraceae (20,61\%), Poaceae $(19,84 \%)$, Fabaceae $(9,16 \%)$, y Verbenaceae $(4,58 \%)$. Muchas familias están pobremente representadas; hay 21 familias con solamente un género y una especie por género. Los géneros mejor representados son: Nasella (4 especies), Schizachyrium (3), Glandularia (3) y Trichocline (3). Solo 19 especies son comunes entre las cuatro combinaciones de tipologías geoquímicas / posiciones altitudinales y se las halló en promedio en el $37 \%$ de los censos.
La endemoflora comprende 37 especies de las cuales 4 son especies endémicas locales (Alternanthera pumila, Apurimacia dolichocarpa, Astragalus parodii, Nassella stuckertii), 16 son endémicas regionales y 17 son endémicas nacionales. Cuatros familias reúnen el $64,8 \%$ de las especies endémicas: Asteraceae (24,32\%), Poaceae $(18,91 \%)$, Fabaceae $(16,21 \%)$ y Verbenaceae $(5,4 \%)$. Entre las especies endémicas regionales que crecen en los afloramientos se destacan: Hyaloseris cinerea var. tomentella, Dalea elegans, Flourensia oolepis, Sphaeralcea cordobensis y Trichocline plicata. Las especies endémicas nacionales con mayor frecuencia son: Thymophylla pentachaeta, Plantago argentina, Gutierrezia gilliesii y Trichocline sinuata. En los afloramientos dolomíticos se destacan como endémicas Astragalus parodii, y Nassella stuckertii y en los afloramientos calcíticos, Polygala stenophylla, Alternanthera pumila, Borreria eryngioides var. ostenii, Polygala stenophylla, Thymophylla pentachaeta, Trichocline plicata. En el grupo de las endémicas regionales algunas especies crecen solo en Córdoba y una o dos provincias limítrofes, así por ejemplo, con San Luis (Trichocline plicata, Gymnocalycium monvillei ssp. monvillei y Nassella hunzikeri), La Rioja y San Luis (Baccharis aliena), Santiago del Estero y San Luis (Sphaeralcea cordobensis), Catamarca y San Luis (Flourensia oolepis).

El total de especies medicinales relevadas en los afloramientos fue de 47. En promedio, un tercio $(33 \%)$ de las especies que crecen en cada afloramiento tiene uso medicinal lo que marca su gran importancia como reservorios de este grupo de plantas. Los afloramientos coincidieron en el valor de taxones medicinales. Entre las especies medicinales endémicas regionales más frecuentes se destacaron Trichocline plicata y Flourensia oolepis, y recíprocamente las menos frecuentes Buddleja cordobensis, Sphaeralcea cordobensis y Baccharis flabellata.

La única especie exótica que crece en estos afloramientos fue Boerhavia diffusa var. diffusa (Nyctaginaceae). El número de especies en los diferentes clados presentes en los afloramientos (Tabla 2) fue mayor en los dominios geoquímicos dolomíticos y también en los localizados en las posiciones más bajas del gradiente.

Las formas de vida más importantes de estos afloramientos fueron las hierbas perennes siempre 


\section{J. J. Cantero et al. - Vegetación de afloramientos carbonáticos del centro de Argentina}

Tabla 2. Clados (sensu APG III, 2009) presentes en los afloramientos de las Sierras de Córdoba.

\begin{tabular}{|ccccccc|}
\hline Afloramientos & Monilófitas & Gimnospermas & Monocotiledóneas & Rósidas & Astéridas & TOTAL \\
\hline$C a+C b$ & 3 & 0 & 25 & 29 & 36 & 93 \\
$D a+D b$ & 3 & 1 & 22 & 28 & 44 & 98 \\
$C a+D a$ & 5 & 1 & 19 & 20 & 31 & 76 \\
$C b+D b$ & 1 & 1 & 23 & 25 & 42 & 92 \\
\hline
\end{tabular}

verdes (46 especies), graminoides (25 especies), hierbas perennes caducifolias (19 especies) y arbustos (8 especies) y las menos representadas son los árboles, hierbas anuales, enredaderas, cactáceas y helechos. No se registraron especies de Bromeliaceae, tal como ocurre en otros afloramientos rocosos (Apéndice).

En la Tabla 3 se muestran los valores indicadores (I.V.) más significativos de taxones que crecen en los dos tipos de afloramientos y diferentes estratos altitudinales. El número de presencias significativas de especies indicadoras fue similar entre los distintos afloramientos y entre estratos, los 31 taxones más representativos con presencias exclusivas se distribuyeron de la siguiente manera:

Da: Astragalus parodii, Danthonia cirrata, Koeleria kurtzii, Margyricarpus pinnatus, Nassella stuckertii, Plantago brasiliensis, Selaginella peruviana, Chascolytrum paleapiliferum.

Ca: Croton argentinus, Sisyrinchium micranthum ssp.valdivianum, Nassella hunzikeri, Plantago argentina, Porophyllum obscurum, Thelesperma megapotamicum.

Cb: Borreria eryngioides var. ostenii, Bouteloua curtipendula var. caespitosa, Hedeoma multiflorum, Polygala stenophylla, Schizachyrium spicatum, Sorghastrum pellitum, Thymophylla pentachaeta, Trichocline plicata.

Db: Adesmia incana var. incana, Bonamia sericea var. sericea, Alternanthera pumila, Dichondra sericea var. holosericea, Paspalum humboldtianum, Phacelia pinnatifida, Polygala subandina, Schizachyrium salzmannii var. aristatum, Selaginella sellowii.

Las comparaciones de los atributos composicionales estructurales de la vegetación entre las diferentes litologías y altitudes revelaron diferencias significativas en diferentes combinaciones: $D a$ vs $D b, C a$ vs $C b, D a$ vs $C a$ y
$D b$ vs $C b$, es decir en los contrastes entre litologías similares en diferentes estratos altitudinales y los contrastes entre diferentes litologías para un mismo estrato altitudinal (Tabla 4). Tanto la altitud como la geoquímica aparecieron asociados a las diferencias observadas en estructura de la vegetación de todos los afloramientos.

Relaciones entre la composición florística, la geoquímica de las rocas y altitud

Por las características de la corta longitud del gradiente $(2,95)$ se asumió que las relaciones entre la vegetación y las variables ambientales estudiadas eran posiblemente de tipo lineal y no unimodales (ter Braak, 2002) y que la variable espacial altitud podía estar enmascarando las asociaciones con la geoquímica de las rocas. Se procedió entonces a aplicar un método de análisis que tiene como modelo subyacente el de relaciones lineales entre la variación de la composición florística y las variables ambientales: el Análisis de Redundancia; se ejecutó primero con la altitud y la geoquímica como variables de interés y posteriormente con la altitud como covariable.

En el triplot de la Fig. 3 se observa cómo se estructura el patrón de la variación florística de los afloramientos con las variables seleccionadas. Para la escala estudiada este patrón aparece asociado al gradiente geoquímico y a la altitud que muestra una posición ortogonal a las variables geoquímicas. Los cuatro afloramientos aparecen claramente diferenciados en diferentes cuadrantes del triplot pero no es posible decidir si las diferencias se deben a la altitud o la geoquímica o a una combinación de ambas.

Los valores de los coeficientes de correlación entre la variable espacial y las ambientales y los ejes del triplot proveniente del RDA (Tabla 5, Fig. 3) sugieren que el primer eje estuvo asociado con altitud y el segundo eje con ambas variables geoquímicas. En el plano del ordenamiento del 
Tabla 3. Análisis de Especies Indicadoras (McCune \& Medford, 1999) de los afloramientos de las Sierras de Córdoba. I.V.valor del indicador (\% de indicación perfecta de cada especie para cada grupo en base a la combinación de las abundancias relativas y frecuencias relativas);

$\mathrm{P}$, proporción de pruebas aleatorizadas con

el valor indicador igual o superior al valor

indicador observado; Frecuencia, frecuencia relativa, porcentaje de cada especie en cada grupo definido por su composición química. Abreviaturas de las especies según Apéndice.

\begin{tabular}{|c|c|c|c|c|c|c|}
\hline \multirow[t]{2}{*}{ Especie } & \multirow[t]{2}{*}{ I. V. } & \multirow[t]{2}{*}{$\mathbf{P}$} & \multicolumn{4}{|c|}{$\begin{array}{l}\text { Frecuencia (\%) en } \\
\text { los Afloramientos }\end{array}$} \\
\hline & & & $D a$ & $\mathrm{Ca}$ & $D b$ & $C b$ \\
\hline Adesinca & 16,7 & 0,008 & 0 & 0 & 17 & 0 \\
\hline Altepumi & 33,3 & 0,002 & 0 & 0 & 0 & 33 \\
\hline Astrparo & 42,6 & 0,000 & 43 & 0 & 0 & 0 \\
\hline Bonaseri & 13 & 0,016 & 0 & 0 & 13 & 0 \\
\hline Borreryn & 14,3 & 0,004 & 0 & 0 & 0 & 14 \\
\hline Boutcurt & 31 & 0,000 & 0 & 0 & 0 & 31 \\
\hline Chaspale & 18,5 & 0,001 & 19 & 0 & 0 & 0 \\
\hline Crotarge & 48,1 & 0,000 & 0 & 48 & 0 & 0 \\
\hline Dantcirr & 33,3 & 0,000 & 33 & 0 & 0 & 0 \\
\hline Dichseri & 51,9 & 0,000 & 0 & 0 & 52 & 0 \\
\hline Hedemult & 14,3 & 0,007 & 0 & 0 & 0 & 14 \\
\hline Koelkurt & 16,7 & 0,002 & 17 & 0 & 0 & 0 \\
\hline Margpinn & 27,8 & 0,000 & 28 & 0 & 0 & 0 \\
\hline Nasshunz & 24,1 & 0,000 & 0 & 24 & 0 & 0 \\
\hline Nassstuc & 59,3 & 0,000 & 59 & 0 & 0 & 0 \\
\hline Pasphumb & 24,1 & 0,001 & 0 & 0 & 24 & 0 \\
\hline Phacepin & 16,7 & 0.0102 & 0 & 0 & 17 & 0 \\
\hline Physamen & 82,5 & 0,000 & 6 & 85 & 0 & 0 \\
\hline Planarge & 22,2 & 0,000 & 0 & 22 & 0 & 0 \\
\hline Planbras & 68,5 & 0,000 & 69 & 0 & 0 & 0 \\
\hline Polysten & 50 & 0,000 & 0 & 0 & 0 & 50 \\
\hline Polysuba & 87 & 0,000 & 0 & 0 & 87 & 0 \\
\hline Poroobsc & 92,6 & 0,000 & 0 & 93 & 0 & 0 \\
\hline Schisalz & 96,3 & 0,000 & 0 & 0 & 96 & 0 \\
\hline Schispic & 57,1 & 0,000 & 0 & 0 & 0 & 57 \\
\hline Selaperu & 16,7 & 0.0028 & 17 & 0 & 0 & 0 \\
\hline Selasell & 37 & 0,000 & 0 & 0 & 37 & 0 \\
\hline Sisyamic & 22,2 & 0,000 & 0 & 22 & 0 & 0 \\
\hline Sorgpell & 19 & 0,001 & 0 & 0 & 0 & 19 \\
\hline Thelmega & 27,8 & 0,000 & 0 & 28 & 0 & 0 \\
\hline Thympent & 66,7 & 0,000 & 0 & 0 & 0 & 67 \\
\hline Tricplic & 31 & 0,000 & 0 & 0 & 0 & 31 \\
\hline
\end{tabular}

RDA (Fig. 4) se pueden observar la importancia de las variables geoquímicas seleccionadas y la variable espacial Altura para cada afloramiento estudiado.

Los afloramientos localizados en las posiciones de mayor altitud, Ca (Los Cienegueros) y Da (Piedra Sonadora) aparecen claramente asociados a la variable Altura en el primer y cuarto cuadrante del triplot (Fig. 3). Sin embargo las tendencias de máxima variación en la abundancia de las especies indicadoras no muestran congruencia con lo hallado en el análisis de las especies indicadoras (Tabla 3). En cambio, para los afloramientos localizados en las altitudes menores, La Argentina (calcítico) y Cuchi Yaco (dolomítico), que aparecen bien diferenciados en los cuadrantes dos y tres respectivamente, hay una total congruencia con la dirección de variación en la abundancia de las especies indicadoras.

Como la variable espacial Altura gobernaba aparentemente una parte importante del patrón de la variación florística, se ejecutó un Análisis de Redundancia parcial (Ter Braak, 2002), donde se consideró a Altura como covariable y a las dos variables geoquímicas como las variables de interés. El examen del triplot de la Fig. 5 sugiere que el primer eje se asoció a $M g O(\mathrm{r}=-0,99)$ quien diferenció a los afloramientos sobre yacencias dolomíticas y el segundo eje a las yacencias calcíticas $(\mathrm{r}=0,39)$. Estas dos variables separaron muy bien las dos tipologías químicas dominantes de los afloramientos hacia la dirección de máxima variación del contenido en óxido de Magnesio en el primer y cuarto cuadrante ( $D b$ y $D a)$ de los afloramientos de rocas con predominio de óxidos de Calcio en su composición, en el segundo y tercer cuadrante $(\mathrm{Ca}$ y $\mathrm{Cb})$ respectivamente.

En el triplot (Fig. 5) se revela además un patrón interesante de congruencia en la variación de abundancia de las especies indicadoras: Bonaseri, Dichseri, Adesinca, Pasphumb, Sellsell, Polysubu y Schisalz, todas indicadoras de afloramientos dolomíticos (Tabla 3), se asociaron a los valores más altos del óxido de Magnesio; en cambio y recíprocamente, Dantcirr, Schispic, Hedemult, Altepumi, Thympent, Thelmega, Polysten, Sorgpell, Planarge y Tricplic (indicadoras de afloramientos cálcicos) lo hicieron con la dirección de máxima variación en la composición del óxido de Calcio. 
J. J. Cantero et al. - Vegetación de afloramientos carbonáticos del centro de Argentina

Tabla 4. Prueba de comparaciones múltiples de medias (Prueba de Tuckey) para Riqueza

$(S)$, diversidad $(H)$, equitatividad $(E)$ y dominancia ( $\left.D^{\prime}\right)$ de los afloramientos de las Sierras de Córdoba.

\begin{tabular}{|c|c|c|c|c|}
\hline & Riqueza & Equitatividad & Diversidad & Dominancia \\
\hline$D a$ & $15,17 \pm 3,26 a$ & $0,96 \pm 0,01 a$ & $2,59 \pm 0.21 \mathrm{a}$ & $0,92 \pm 0,02 a$ \\
\hline$D b$ & $13,70 \pm 2,70 b$ & $0,95 \pm 0,94 b$ & $2,48 \pm 0.20 b$ & $0,90 \pm 0,02 b$ \\
\hline $\mathrm{Ca}$ & $13,28 \pm 3,25 a$ & $0,94 \pm 0,02 \mathrm{a}$ & $2,42 \pm 0,25 a$ & $0,89 \pm 0,03 a$ \\
\hline$C b$ & $16,79 \pm 3,42 b$ & $0,96 \pm 0,02 b$ & $2,69 \pm 0,25 b$ & $0,92 \pm 0,03 b$ \\
\hline$D a$ & $15,17 \pm 3,26 b$ & $0,96 \pm 0,01 b$ & $2,59 \pm 0,21 b$ & $0,92 \pm 0,02 b$ \\
\hline $\mathrm{Ca}$ & $13,28 \pm 3,25 a$ & $0,94 \pm 0,02 \mathrm{a}$ & $2,42 \pm 0,25$ a & $0,89 \pm 0,03 a$ \\
\hline$D b$ & $13,70 \pm 2,70 a$ & $0,95 \pm 0.01 a$ & $2,48 \pm 0.20 a$ & $0,90 \pm 0,02 a$ \\
\hline$C b$ & $16,79 \pm 3,42 b$ & $0,96 \pm 0.02 b$ & $2,70 \pm 0.25 b$ & $0,92 \pm 0,03 b$ \\
\hline $\mathrm{Ca}$ & $13,28 \pm 3,25 a$ & $0,94 \pm 0,02$ a & $2,42 \pm 0,25 a$ & $0,89 \pm 0,03 a$ \\
\hline$D b$ & $13,70 \pm 2,70 a$ & $0,95 \pm 0,01 b$ & $2,48 \pm 0,20 a$ & $0,90 \pm 0,02 a$ \\
\hline $\mathrm{Cb}$ & $16,79 \pm 3,42 b$ & $0,96 \pm 0,02 a$ & $2,70 \pm 0,25 b$ & $0,92 \pm 0,03 a$ \\
\hline$D a$ & $15,17 \pm 3,26 a$ & $0,96 \pm 0,01 \mathrm{a}$ & $2,59 \pm 0,21$ a & $0,92 \pm 0,02 a$ \\
\hline
\end{tabular}

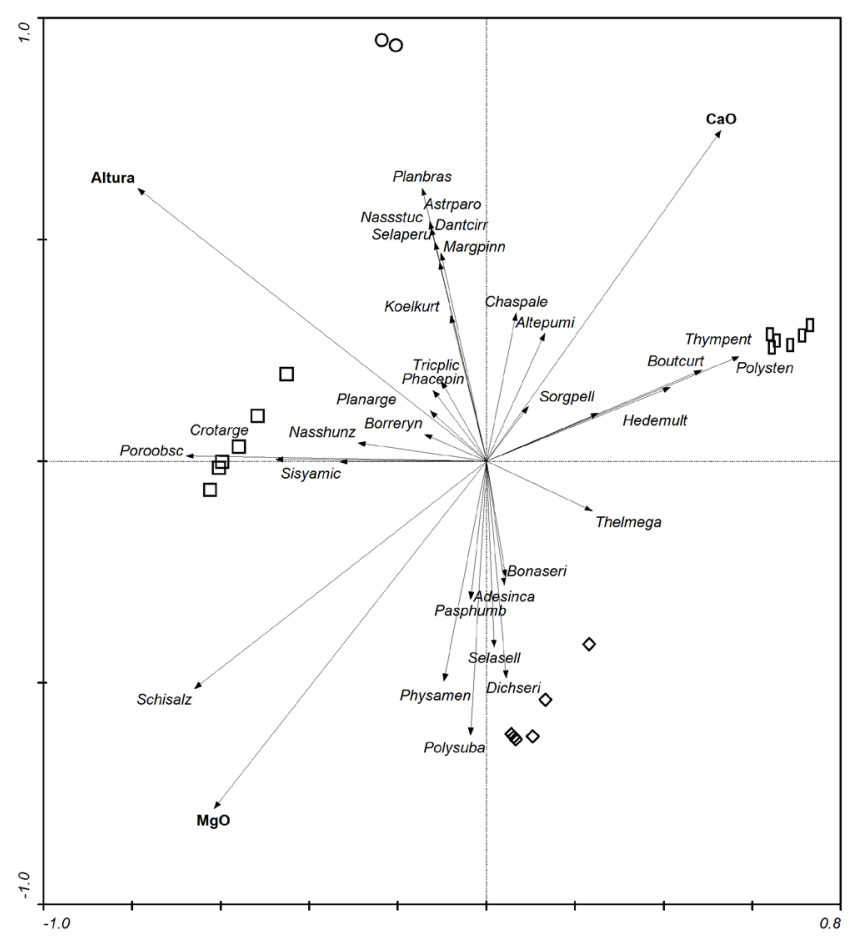

Fig. 3. Análisis de Redundancia (RDA), triplot con (1) afloramientos (Ca, Cálcicos altos, círculos; $D a$, Dolomíticos altos, cuadrados; $D b$, Dolomíticos bajos, rombos y $C b$, Cálcicos bajos, rectángulos), y (2) variables ambientales: altura sobre el nivel del mar (Altura), óxido de calcio ( $\mathrm{CaO})$, óxido de magnesio $(\mathrm{MgO})$. Eigenvalue eje 1, 0,16; eje 2, 0,10; correlación (r) especies-ambiente: eje 1, 0,94; eje 2, 0,88; $27 \%$ de la variación florística y $90 \%$ de la relación vegetación-ambiente explicada por los dos primeros ejes. Prueba de Monte Carlo significativa $(P<0,01)$. Suma de los valores propios canónicos, 0,30 . Los denominación de los grupos de censos son: círculos, $C a$; cuadrados, $\mathrm{Da}$; rombos, $\mathrm{Db}$; rectángulos, Cb. Las especies (indicadoras) se corresponden con las de la Tabla 3. Abreviaturas de las especies según Apéndice. 
Bol. Soc. Argent. Bot. 49 (4) 2014

A

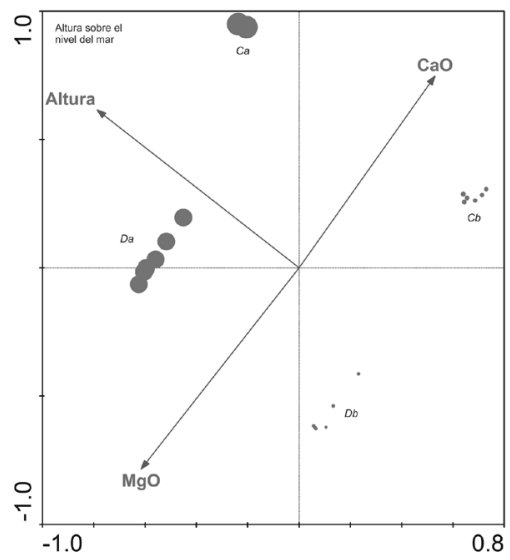

B
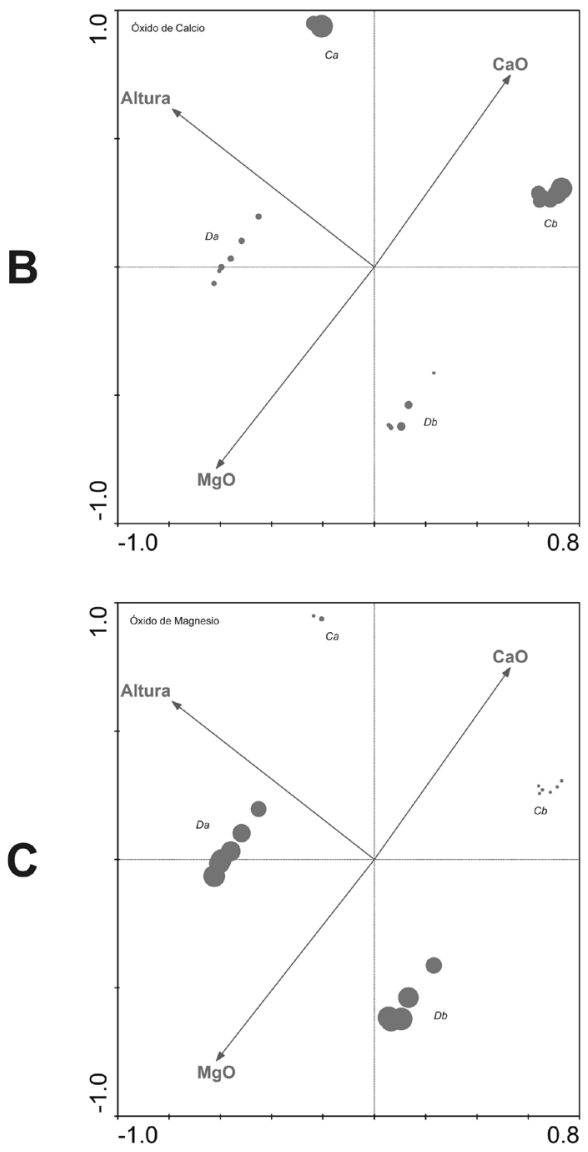

Fig. 4. Variables en el plano del ordenamiento del RDA. A: Variable espacial (Altura). B-C: Variables geoquímicas ( $\mathrm{CaO}, \mathrm{MgO}$, respectivamente). Los centros de los círculos están localizados en las posiciones de los respectivos afloramientos en el espacio del ordenamiento. El tamaño de los círculos se corresponde con el valor de la variable observada para cada sitio en particular.
Tabla 5. Coeficientes de correlación

de Pearson de la variable espacial y

geoquímicas con los dos primeros ejes del ordenamiento generado por RDA (Fig. 3).

\begin{tabular}{|lcc|}
\hline & Eje 1 & Eje 2 \\
\hline Oxido de Calcio & 0,53 & 0,75 \\
Oxido de Magnesio & $-0,61$ & $-0,78$ \\
Altura & $-0,78$ & 0,61 \\
\hline
\end{tabular}

\section{Discusión}

La flora de los afloramientos carbonáticos estudiados, de baja riqueza y diversidad, está dominada por eudicotiledóneas nativas perennes de larga vida, especialmente hierbas, graminoides y arbustos, tal como ocurre, para una escala mayor, en el Distrito fitogeográfico al que pertenecen (Giorgis et al., 2011). El clado de las Monocotiledóneas está básicamente representado por las Poaceae que son importantes codominantes de la composición florística. La magnitud de los diferentes clados fue siempre mayor en los dominios geoquímicos dolomíticos tanto en la escala regional de los afloramientos (Cantero et al., 2011) como en la escala local estudiada en este trabajo.

Las tres familias más numerosas de los afloramientos, Asteraceae, Poaceae, Fabaceae, son también las mismas que caracterizan con esas magnitudes al Distrito Chaqueño Serrano (Giorgis et al., 2011). Sin embargo el orden de importancia de esas familias y sus proporciones respecto a la flora total, son diferentes: en los afloramientos la más importante es Asteraceae, en cambio en el Distrito, son las Poaceae, y las proporciones de los tres taxones fueron siempre mayores en los afloramientos. En el caso de los géneros más numerosos, no hay ninguna coincidencia en los mismos, aspecto que seguramente se asocia a la especificidad del hábitat estudiado en este trabajo. Del total de la flora relevada en los afloramientos carbonáticos hay 19 especies que no están presentes en el catálogo de Giorgis et al. (2011), entre ellas, algunas importantes indicadoras de las diferentes geoquímicas (Junellia bisulcata var. campestris, Physaria mendocina, Trichocline sinuata, entre otras).

Las especies se distribuyen a lo largo de gradientes ambientales o tipos de hábitat de acuerdo a sus nicho- $\beta$ (Ackerly \& Corwell, 2007), 


\section{J. J. Cantero et al. - Vegetación de afloramientos carbonáticos del centro de Argentina}

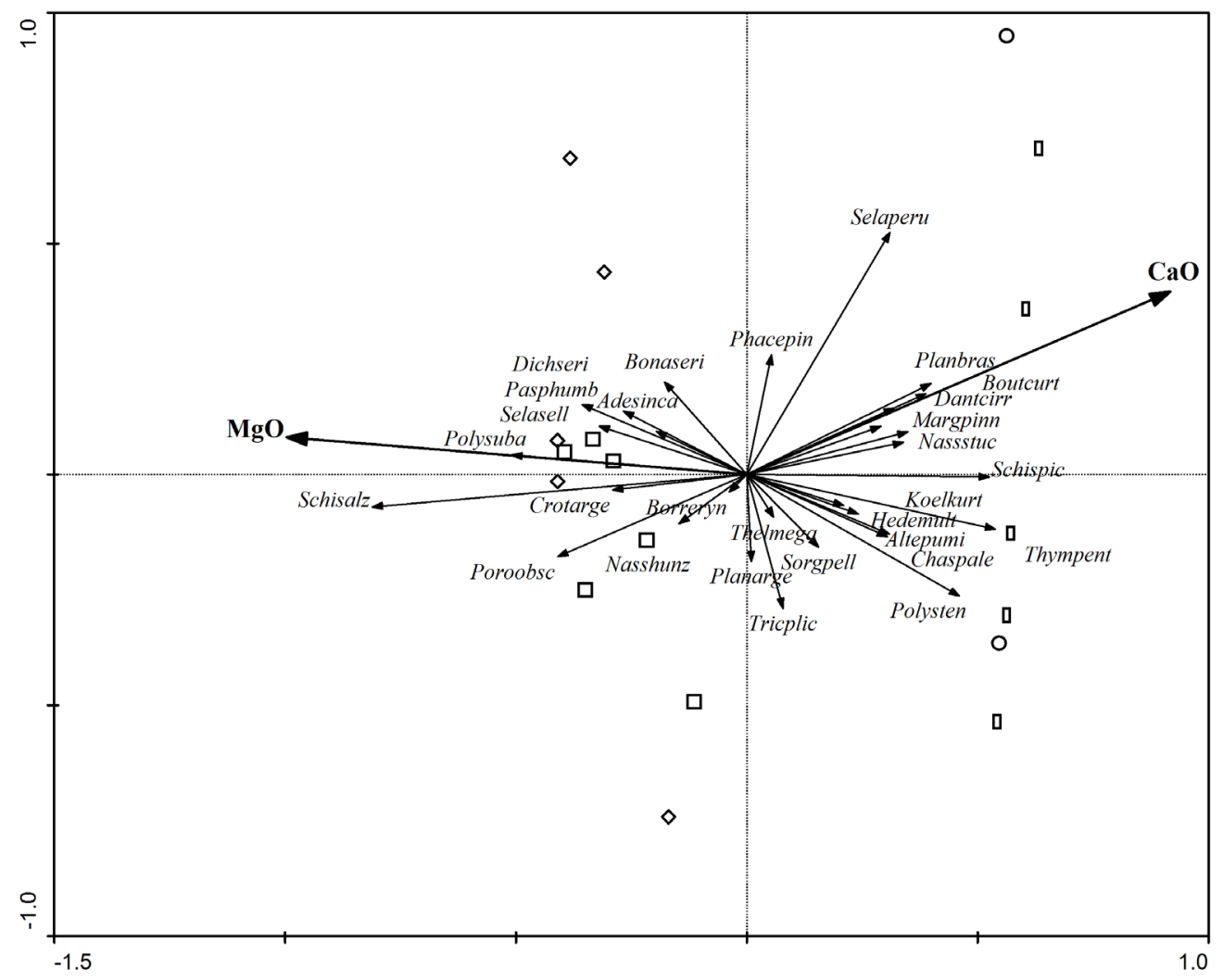

Fig. 5. Análisis de Redundancia parcial (partial-RDA), triplot con (1) afloramientos (ref. idem Fig. 3), y (2) variables ambientales: óxido de calcio $(\mathrm{CaO})$ y óxido de magnesio $(\mathrm{MgO})$. Eigenvalue eje 1, 0,13; eje 2, 0,03; correlación ( $r$ ) especies-ambiente: eje 1, 0,90; eje 2, 0,63; $33 \%$ de la variación florística y $100 \%$ de la relación vegetación-ambiente explicada por los dos primeros ejes. Prueba de Monte Carlo significativa $(P<0,002)$. Suma total de los eigenvalues, 0,86 ; suma de los eigenvalues canónicos luego del ajuste de la covariable, 0,16 . Las especies (indicadoras) se corresponden con las de las Tabla 3. Abreviaturas de las especies según Apéndice.

resultando en una coexistencia de comunidades más que de especies. Para la coexistencia de especies es más importante el nicho- $\alpha$ (Silvertown et al., 2006). Los estudios en gran escala usualmente abarcan diferentes tipos de vegetación (pastizales, arbustales, bosques) que deberían ser analizados en forma separada (Tamme et al., 2010). Los estudios de escala regional realizados en el Distrito del Chaco Serrano por Cantero et al. (2011) y Giorgis et al. (2011) involucraron diferentes tipos de vegetación (bosques, arbustales y pastizales) que crecían en diferentes tipos de afloramientos (mármoles cálcicos, mármoles dolomíticos y serpentinitas) y es posible que esos tipos respondan a la heterogeneidad en diferentes escalas espaciales (Schenk \& Jackson, 2002).
Cada comunidad tiene su propio pool de especies, es decir el grupo de especies que pueden potencialmente acceder y crecer en la misma (Eriksson, 1993; Zobel, 1997). La fracción ausente del pool, para cualquier momento de tiempo, es llamada diversidad oscura (Pärtel et al., 2011). Ser miembro de un pool de especies demanda que exista una coincidencia entre los requerimientos de hábitat de la especie (nicho $\beta$, nicho de hábitat) y las condiciones ambientales particulares de la comunidad local bajo estudio. Como resultado, el pool de especies es hábitat-específico, por lo tanto diferentes tipos de comunidades dentro de una misma región poseen también diferentes pool de especies. Solo dos números, el número de especies actualmente presentes y el número de 
especies potencialmente presentes, determinan la completitud de una determinada comunidad (Pärtel et al., 2013). En relación a los dos dominios geoquímicos carbonáticos a nivel regional en las Sierras de Córdoba, el calcítico y el dolomítico, el pool de especies de la vegetación del primero está integrado por 35 especies y el pool de especies del segundo por 49 especies (Cantero et al., 2011). $\mathrm{Si}$ bien la información disponible es insuficiente para poder calcular la métrica de completitud en la forma como lo plantea Pärtel et al. (2013), siguiendo su concepto se podría plantear en forma preliminar que la diversidad oscura es baja en los cuatro afloramientos estudiados ya que está representado el $71,42 \%$ del pool regional de los afloramientos calcíticos y el $59,18 \%$ de los dolomíticos respectivamente.

Los sitios estudiados son aparentemente importantes filtros ambientales a las invasiones por plantas exóticas (Fleischmann, 1997; Meirelles et al., 1999) ya que una sola especie, Boerhavia diffusa var. diffusa, tiene carácter adventicio en la flora relevada. Además resguardan, a manera de refugio (Gram et al., 2004; Speziale \& Ezcurra, 2010), a un grupo muy importante de plantas endémicas.

$\mathrm{Al}$ igual que afloramientos de otros continentes (Burke, 2002a, b; Wiser \& Buxton, 2008), los afloramientos carbonáticos no tienen estrictamente un carácter insular ya que comparten especies con la matriz circundante, tanto a lo largo del tiempo evolutivo como del tiempo ecológico. Sin embargo, en ellos crecen especies muy singulares por su fidelidad, un hecho común en yacencias de estas características (Stevanovic et al., 2003; Safford et al., 2005; Selvi, 2007; Baskin \& Baskin, 2007), así por ejemplo dos especies endémicas Junellia bisulcata var. campestris y Thymophylla pentachaeta son exclusivas de los afloramientos $\mathrm{y}$ no se han encontrado creciendo en la matriz circundante (González et al., 1998, 1999; Cantero et al., 1999, 2001; González et al., 2002; Núñez et al., 2002; Petryna et al., 2002; Cantero et al., 2003; Goleniowski et al., 2006).

Las relaciones entre la heterogeneidad espacial ambiental y la diversidad de la especies es uno de los principales conceptos en ecología de comunidades, sin embargo no ha sido estudiada profundamente (Wilson, 2000). La heterogeneidad espacial es definida por Ettema \& Wardle (2002) como la variabilidad en la estructura espacial, es decir que la distribución espacial no es al azar o uniforme sino agregada o irregular. En general se acepta que los ambientes más heterógeneos pueden sostener más especies que los homogéneos, sin embargo la evidencia empírica es escasa (Lundholm, 2009) y existen además estudios que muestran relaciones negativas (Tamme et al., 2010). Los factores que provocan heterogeneidad varían de acuerdo a la escala espacial (Pickett et al., 2000; Ettema \& Wardle, 2002). Lundholm (2009) reconoce diferentes parámetros de la escala espacial: extensión, grano y escala focal. La heterogeneidad abiótica es causada principalmente por el clima, topografía local o material originario (Hodge, 2005) y por lo tanto ocurre en escalas espaciales relativamente grandes (Pickett et al., 2000) y allí la heterogeneidad se expresa principalmente como gradientes de factores ambientales. En cambio, la heterogeneidad biótica es causada típicamente por microorganismos de suelo (Ettema \& Wardle, 2002), herbívoros (Augustine \& Frank, 2001) o por la absorción y deposición irregular de recursos por las plantas (Wilson, 2000), es decir en escalas espaciales más pequeñas (Tamme et al., 2010).

Para una escala grande (regional), tanto en extensión (área total de estudio $33.600 \mathrm{~km}^{2}$, tres tipos geoquímicos contrastantes de rocas y de vegetación), grano (presencia/ausencia de especies) y escala focal $\left(2725 \mathrm{~m}^{2}\right)$, Cantero et al. (2011) encontraron que el gradiente geográfico y el gradiente altitudinal se asociaban estrechamente con el patrón florístico de 404 especies que crecían en 31 afloramientos con una composición diferencial en $\mathrm{Ca}$ y $\mathrm{Mg}$ (mármoles cálcicos, mármoles dolomíticos, serpentinitas y travertinos). La variación ambiental a nivel regional, variable a lo largo del gradiente geográfico (y climático), se sobrepuso y enmascaró la variación local ambiental (geoquímica, altitud) entre los afloramientos.

En este trabajo, la escala fue diferente (local), tanto en extensión (área total de estudio $412 \mathrm{~km}^{2}$, dos tipos geoquímicos contrastantes de rocas y un único tipo de vegetación), grano (abundanciacobertura de las especies), y escala focal $\left(1 \mathrm{~m}^{2}\right)$. Para esta escala más pequeña y más homogénea (un único tipo de vegetación) la riqueza promedio y el número de especies endémicas halladas fueron previsiblemente menores respecto a la escala regional, 68 a 14 y 88 a 57 respectivamente. Si 


\section{J. J. Cantero et al. - Vegetación de afloramientos carbonáticos del centro de Argentina}

bien la riqueza regional de endemismos estuvo relacionada positivamente con el área total de los afloramientos muestreada (área específica de afloramientos $0,93 \mathrm{~km}^{2}$ en la escala regional vs. $0,26 \mathrm{~km}^{2}$ en la escala local), tal como ha sido hallado en otros estudios (Harrison et al., 2006), el cambio de escala tuvo un impacto mayor en la riqueza florística total que en la endemoflora.

La evaluación exploratoria inicial de las asociaciones vegetación-ambiente mostró a los grupos de censos fuertemente ensamblados de acuerdo tanto a su posición en el gradiente altitudinal como a la tipología geoquímica dominante de las rocas subyacentes. Es decir, para una escala local, la altitud siguió siendo un factor espacial importante en la explicación de la variación en la composición florística. La metodología empleada permitió considerar la influencia de la geoquímica sobre la variación composicional luego de haber eliminado el efecto de la altitud y el patrón emergente reveló una estrecha asociación entre la composición de la vegetación y la dominancia de los óxidos de $\mathrm{Ca}$ y de $\mathrm{Mg}$ (ambos siempre presentes) en las rocas asociadas.

Si bien es este trabajo no se consideraron suficientes réplicas como para poder establecer conclusiones contundentes, es posible, reconociendo el efecto primario de la altitud, establecer relaciones entre la presencia de diferentes taxones de plantas vasculares y tipologías geoquímicas (calcítica y dolomítica) de manera independiente a su posición en el gradiente de altitud, confirmándose la importancia que tienen estas variables en la estructuración del hábitat y filtrado abiótico de especies. Efectos combinados de la geoquímica y la altitud sobre la composición florística ha sido reconocida en diferentes afloramientos (Wiser et al., 1996; Chiarucci et al., 2001; Grace et al., 2007; Tsiripidis et al., 2010). En afloramientos de serpentinitas de USA, usando una metodología de análisis más sofisticado (SEM, modelación de ecuaciones estructurales), Harrison et al. (2006) hallaron también una correlación importante entre los patrones de variación florística y las variables ambientales explicativas para ambas escalas, regional y local. Para conservar la vegetación singular de los afloramientos carbonáticos centroargentinos, y teniendo en cuenta la completitud de sus comunidades integrantes y la asociación compleja con factores espaciales y ambientales sería importante proteger una red de afloramientos que representen el amplio rango de la variación geológica, geográfica y altitudinal de los mismos.

\section{Agradecimientos}

Este trabajo fue financiado por la Secretaría de Ciencia y Tecnología de la Universidad Nacional de Río Cuarto y la Secretaría de Minería de la Provincia de Córdoba.

\section{Bibliografía}

ACKERLY, D.D. \& W.K. CORWELL. 2007. A traitbased approach to community assembly: partitioning of species trait values into within -and amongcommunity components. Ecol. Lett.10: 135-145.

AGUILAR, R. \& L. GALETTO. 2004. Effects of forest fragmentation on male and female reproductive success in Cestrum parqui (Solanaceae). Oecologia 138: 513-520.

AIZEN, M.A. \& P. FEINSINGER. 1994a. Forest fragmentation, pollination, and plant reproduction in a Chaco dry forest, Argentina. Ecology 75: 330-351.

AIZEN, M.A. \& P. FEINSINGER. 1994b. Habitat fragmentation, native insect pollinators, and the feral honey bees in argentine Chaco Serrano. Ecol. Appl. 4: 378-392.

ALVES, R. \& J. KOLBEK. 1994. Plant-species endemism in savanna vegetation on Table Mountains (camporupestre) in Brazil. Vegetatio 113: 125-139.

ANDERSON, R.C., J. S. FRALISH \& J. M. BASKIN. 1999. Introduction. In: R. C. ANDERSON, J. S. FRALISH \& J. M. BASKIN (eds.), Savannas, barrens, and rock outcrop plant communities of North America, pp.1-4. Cambridge University Press, Cambridge.

ASHTON, D. H. \& R. N. WEBB.1977. The ecology of granite outcrops at Wilson's Promontory, Victoria. Austral. J. Ecol. 2: 269-296.

AUGUSTINE, D.J \& D.A. FRANCK. 2001. Effect of migratory grazers on spatial heterogeneity of soil nitrogen properties in a grassland ecosystem. Ecology 82: 3149-3162.

BARBOZA, G. E., J. J. CANTERO, C. O. NÚÑEZ \& L. ARIZA ESPINAR (eds.). 2006. Flora medicinal de la Provincia de Córdoba. Pteridófitas y Antófitas silvestres o naturalizadas. Museo Botánico, Córdoba, Argentina.

BARBOZA, G. E., J. J. CANTERO, C. O. NÚÑEZ, A. PACCIARONI \& L. ARIZA ESPINAR. 2009. Medicinal plants: a general review, a phytochemical, 
and ethnopharmacological screening of the native Argentine Flora. Kurtziana 34: 7-365.

BARThlotT, W. A., A. GREGER \& A. POREMBSKI.1993. Some remarks on the vegetation of tropical inselbergs: Diversity and ecological differentiation. Biogegraphica 69: 106-124.

BASKIN, J.M. \& C.C.BASKIN. 1988. Endemism in rock outcrop plant communities of unglaciated eastern United States: an evaluation of the roles of the edaphic, genetic and light factors. J. Biogeogr. 15: 829-840.

BIANCHI, A. \& S. CRAVERO. 2010. INTA. Programa Nacional Ecoregiones. República Argentina. Atlas Climático Digital.

BRAUN BLANQUET, J. 1979. Fitosociología. Bases para el estudio de las comunidades vegetales. Blume Ediciones, Madrid.

BURBANCK, M. P. \& R. B. PLATT. 1964. Granite outcrop comunities of the Piedmont Plateau in Georgia. Ecology 45: 292-306.

BURKE, A. 2002a. Island-matrix relationships in Nama Karoo inselberg landscapes Part I: Do inselbergs provide a refuge for matrix species? Plant Ecol. 160: 79-90.

BURKE, A. 2002b. Island-matrix relationships in nama Karoo inselberg landscapes Part II: Are some inselbergs better sources than others? Plant Ecol. 158: 41-48.

BURKE, A., K. J. ESLER, E. PIENAAR \& P. BARNARD. 2003. Species richness and floristic relationships between mesas and their surroundings in southern African Nama Karoo. Divers. Distrib. 9: 43-53.

CABIDO, M., A. ACOSTA \& S. DÍAZ. 1990. The vascular flora and vegetation of granitic outcrops in the upper Córdoba mountains. Argentina. Phytocoenologia 19: 267-281.

CABIDO, M., G. FUNES, E. PUCHETA, F. VENDRAMINI \& S. DÍAZ. 1998. A chorological analysis of the mountains of central Argentina. Is all what we call Sierra Chaco really Chaco? Contribution to the study of flora and vegetation of Chaco. XII. Candollea 53: 321-331.

CABRERA, A. 1976. Regiones fitogeográficas argentinas. 2 ed. Enciclopedia Argentina de Agricultura y Jardinería. ACME, Buenos Aires.

CAGNOLO, L., CABIDO, M. \& G. VALLADARES. 2006. Plant species richness in the Chaco Serrano Woodland from central Argentina: Ecological traits and habitat fragmentation effects. Biol. Conserv. 132: 510-519.

CANTERO, J. J., M. PÄRTEL \& M. ZOBEL. 1999. Is species richness dependent on the neighbourhood? An analysis of the community patterns in mountain grasslands of central Argentina. Oikos 87: 346-355.

CANTERO, J. J., M. CABIDO, C. NUÑEZ,
L. PETRYNA, M. ZAK \& M. ZOBEL. 2001. Clasificación de los pastizales de suelos sobre rocas metamórficas de las Sierras de Córdoba, Argentina. Kurtziana 29: 27-77.

CANTERO, J. J., J. LIIRA, J. M CISNEROS, J. C GONZÁLEZ, C. O. NÚÑEZ, L.PETRYNA, C., CHOLAKY \& M. ZOBEL. 2003. Species richness, alien species and plant traits in central Argentine mountain grasslands. J. Veg. Sci.14: 129-136.

CANTERO, J.J., J. A. SFRAGULLA, C. NÚÑEZ, A. A. BONALUMI, J. MULKO, A. AMUCHASTEGUI, F. CHIARINI, G.E. BARBOZA \& L. ARIZA ESPINAR. 2011. Flora de los afloramientos de mármoles y serpentinitas de las Sierras de Córdoba (Argentina). Kurtziana 36: 11-45.

D’ALOIA, M. 1959. Las calizas y calcáreos de Córdoba. Dirección Provincial de Minería. Córdoba.

CHIARUCCI, A., D. ROCCHINI, C.LEONZIO \& V. DE DOMINICIS. 2001. A test of vegetationenvironment relationship in serpentine soils of Tuscany, Italy. Ecol. Res. 16: 627-639.

DE LANGE P.J., D. A. NORTON, P. B. HEENAN, S. P. COURTNEY, B. P. J. MOLLOY, C. C. OGLE, B. D. RANCE, P. N. JOHNSON \& R. HITCHMOUGH. 2004. Threatened and uncommon plants of New Zealand. New Zealand J. Bot. 42: 45-76.

DI RIENZO J.A., CASANOVES F., BALZARINI M.G., GONZALEZ L., TABLADA M. \& ROBLEDO C.W. InfoStat versión 2011. Grupo InfoStat, FCA, Universidad Nacional de Córdoba.

ERIKSSON O. 1993. The species-pool hypothesis and plant community diversity. Oikos 68: 371-374.

ESCUDERO, A. 1996. Community patterns on exposed cliffs in a Mediterranean calcareous mountain. Vegetatio 125: 99-110.

ESGARIO, C. P., A. P. FONTANA \& A. G. SILVA.2009. A flora vascular sobre rocha no Alto Misterioso, uma área prioritária para a conservação da Mata Atlântica no Espírito Santo, Sudeste do Brasil Natureza on line 7: 80-91. [on line] http://www.naturezaonline. com.br

ETTEMA, C.H. \& D.A.WARDLE. 2002. Spatial soil ecology. Trends Ecol. Evol. 17: 177-183.

FERNÁNDEZ ARECES, M., P. PENAS \& T. DÍAZ. 1983. Aportaciones al conocimiento de las comunidades vegetales de los paredones rocosos calizos de la Cordillera Cantábrica. Anales Jard. Bot. Madrid 40: 213-219.

FLEISCHMANN, K. 1997. Invasion of alien woody plants on the islands of Mah'e and Silhouette, Seychelles. J. Veget. Sci. 8: 5-12.

FUNES, G. \& M. CABIDO. 1995. Variabilidad local y regional de la vegetación rupícola de las Sierras grandes de Córdoba. Kurtziana 24: 173-188.

GALANDE MERA,A., C. CÁCERES \&A. GONZÁLEZ. 


\section{J. J. Cantero et al. - Vegetación de afloramientos carbonáticos del centro de Argentina}

2003. La vegetación de la alta montaña andina del sur del Perú. Acta Bot. Malacitana 28: 121-147.

GIORGIS, M., CINGOLANI, A.M., CHIARINI, F., CHIAPELLA, J., BARBOZA, G., ARIZA ESPINAR, L., MORERO, R., GURVICH, D.E., TECCO, P.A., SUBILS, R. \& CABIDO, M. 2011. Composición florística del Bosque Chaqueño Serrano de la provincia de Córdoba, Argentina. Kurtziana 36: 9-43.

GIULIETTI, A. M., J. PIRANI \& R. M. HARLEY. 1997. Espinhaço range region, eastern Brazil, en: S. D. DAVIS, V. H. HEYWOOD, O. HERRERAMACBRYDE, J. VILLA-LOBOS \& A. C. HAMILTON (eds.), Centres of Plant Diversity: A Guide and Strategy for their Conservation, pp. $397-$ 404. IUCN Publication Unity, v.3, Cambridge.

GOLENIOWSKI, M. E., G. BONGIOVANNI, L.PALACIO, C.O. NÚÑEZ \& J. J. CANTERO. 2006. Medicinal plants from the "Sierra de Comenchingones", Argentina. J. Ethnopharmacol. 107: 342-341.

GONZÁLEZ, J. G., J. J. CANTERO \& J. M. CISNEROS. 1998. Caracterización de la estructura de los paisajes serranos del centro de Argentina. Revista Univ. Nac. Rio Cuarto 18: 25-37.

GONZÁlEZ, J. G., J. J. CANTERO, C. O. NÚÑEZ, C. CHOLAKY, J. M. CISNEROS \& L. PETRYNA. 1999. Cartografía de los ecogrupos vegetales en los paisajes serranos del centro sur de Córdoba, Argentina. Revista Univ. Nac. Río Cuarto 19: 5-17.

GONZÁlEZ, J. G., T. ROBINSON, J. M. CISNEROS \& J. J. CANTERO. 2002. Utilización de los SIG en la gestión de los recursos naturales en una cuenca serrana del centro argentino. Revista Univ. Nac. Río Cuarto 22: 27-35.

GORDILLO, C. \& A. LENCINAS. 1979. Sierras Pampeanas de Córdoba y San Luis. II. Simp.Geol. Reg. Arg. Acad. Nac. Cienc. Córdoba 1: 577-650.

GRACE, J., H. SAVORD \& S. HARRISON. 2007. Large-scale causes of variation in the serpentine vegetation of California. Plant Soil 293: 121-132.

GRAM, W., E. BORER, K. COTTINGHAM, E. SEABLOOM, V. BOUCHER, L. GOLDWASSER, F. MICHELI, B. KENDALL, \& R. BURTON. 2004. Distribution of plants in a California serpentine grassland: are rocky hummocks spatial refuges for native species? Plant Ecol. 172: 159-171.

GUTTE, P. 1986. Beitrag zurkenntnis central-peruanischer Planzengeselleschaften. III. Pflanzengesellschaften der subalpine Stufe. Feddes Repert. 97: 319-371.

HARRISSON, S., K. DAVIES, H. SAFFORD \& J. VIERS. 2006. Beta diversity and the scaledependence of the productivity relationships: a test in the Californian serpentine flora. J. Ecol. 94: 110-117.
HEYWOOD, V. H. 1953. E1 concepto de asociación en las comunidades rupícolas. Anales Inst. Bot. Cavanilles 11: 463-481.

HODGE, A. 2005. Plastic plants and patchy soils. J. Exp. Bot. 57: 401-411.

HUNTER J. T. 2003. Persistence on inselbergs: the role of obligate seeders and resprouters. J. Biogeogr. 30: 497-510.

INTA. 2014. Instituto de Clima y Aguas. http://climayagua. inta.gob.ar/.

JACOBI, C. M., F. CARMO, R. VINCENT \& J. R. STEHMANN. 2007. Plant communities on ironstone outcrops: a diverse and endangered Brazilian ecosystem. Biodivers. Conserv. 16: 2185-2200.

KRUCKEBERG, A. R. 2002. Geology and Plant Life. University of Washington Press, Seattle.

LARSON, D., U. MATTHES \& P. E. KELLY. 2000. Cliff ecology: pattern and process in cliff ecosystems. Cambridge Studies in Ecology. Cambridge University Press, Cambridge.

LUNDHOLM, J.T. 2009. Plant species diversity and environmental heterogeneity: spatial scale and competing hypotheses. J. Veget. Sci. 20: 377-391.

MARTÍNEZ CARRETERO, E. 1994. La vegetación saxícola de la precordillera de Mendoza. VI Congreso Latinoamericano de Botánica, Mar del Plata.

MAYCOCK P. F. \& D. FAHSELT. 1992. Vegetation of stressed calcareous screes and slopes in Sverdrup Pass, Ellesmere Island, Canada. Canad. J. Bot. 70: 2359-2377.

McCUNE, B. \& J.B. GRACE. 2002. Analysis of Ecological Communities. MjM Software, Gleneden Beach, Oregon.

McCUNE, B. \& M. MEFFORD. 1999. Multivariate Analysis of Ecological Data, Version 4.0. MjM Software. Gleneden Beach, Oregon.

MEDINA CAZORLA, J., F. PEREZ GARCIA, J. GARRIDO-BECERRA, F. MARTINEZHERNANDEZ, A. MENDOZA, A. PEREZ LATORRE \& J. MOTA. 2005. Riqueza y rareza florísticas en los afloramientos dolomíticos de las Cordilleras Béticas (sur de España): ensayo preliminar. Acta Bot. Malacitana 30: 111-127.

MEIRELLES, T. S., PIVELLO, V.R \& C.A. JOLY.1999. The vegetation of granite rock outcrops in Rio de Janeiro, Brazil, and the need for its protection. Environ. Conserv. 26: 10-20.

MÉNDEZ, E. 2010. La vegetación saxícola y de taludes del Flanco oriental del Cordón del Plata (Luján de Cuyo, Mendoza, Argentina). Bol. Soc. Argent. Bot. 45: 119-147.

MICHELANGELI, F. A. 2000. Species composition and species-area relationships in vegetation isolates on summit of a sandstone mountain in southern Venezuela. J. Trop. Ecol. 16: 69-82. 
MORAT, P., JAFFRE, T. \& J.-M.VEILLON. 2001. The flora of New Caledonia's calcareous substrates. Adansonia 23: 109-127.

MOTA, J., J. M. MEDINA-CAZORLA, F. BRUNO NAVARRO, J. PÉREZ-GARCÍA，A. PÉREZLATORRE, P. SÁNCHEZ-GÓMEZ, J. TORRES, A. BENAVENTE, G. BLANCA, C. GIL, J. LORITE \& M. E. MERLO.2008. Dolomite flora of the Baetic Ranges glades (South Spain). Flora 203: 359-375.

NÚÑEZ, C.O., J. J. CANTERO, G. RAMÍREZ, L. PETRYNA \& A. AMUCHÁSTEGUI. 2002. Efectos del fuego y pastoreo sobre tipos funcionales de Poáceas C3 y C4 en pastizales serranos centroargentinos. Agrosur (Chile) 30: 12-24.

ORTIZ, S. \& J. RODRÍGUEZ OUBIÑA. 1993. Synopsis of the rupicolous vegetation of Galicia (Northwestern Iberian Peninsula). Folia Geobot. Phytotax. 28: $15-49$.

PETRYNA, L., M. MOORA, C. O. NÚÑEZ, J. J. CANTERO \& M. ZOBEL. 2002. Are invaders disturbance-limited? Conservation of mountain grasslands in central Argentina. Appl. Veget. Sci. 5: 195-202.

PÄRTEL, M.,R. SZAVA-KOVATS\& M. ZOBEL. 2011. Dark diversity: shedding light on absent species. Trends Ecol. Evol. 26: 124 - 128.

PÄRTEL, M., R. SZAVA-KOVATS \& M. ZOBEL. 2013. Community completeness: linking local and dark diversity within the species pool concept. Folia Geobot. 48: 307-317.

PICKETT, S. T. A., M. L. CADENASSO \& C.G. JONES. 2000. Generation of heterogeneity by organisms: creation, maintenance and transformation. In: M. HUTCHINGS, E. A. JOHN \& A.J.A. STEWART (eds.), The ecological consequences of environmental heterogeneity, pp. 33-52. Blackwell, Oxford.

POPE, N., H. TANNER \& N. RAJAKARUNA. 2010. Vascular plants of adjacent serpentine and granite outcrops on the deer isles, Maine, U.S.A. Rhodora 112 (950): 105-141.

POREMBSKI, S., W. BARTHLOTT, S. DÖRRSTOCK \& N. BIEDINGER. 1994. Vegetation of rock outcrops in Guinea: granite inselbergs, sandstone table mountains and ferricretes - remarks on species numbers and endemism. Flora 189: 315-326.

RAUNKIAER, C. 1934. Life forms and terrestrial plant geography. Clarendon Press, Oxford.

ROGERS G. \& S. WALKER. 2002. Taxonomic and ecological profiles of rarity in the New Zealand vascular flora. New Zealand J. Bot. 40: 73-93.

SADLER, K. D. \& G. E. BRADFIELD. 2010. Ecological facets of plant species rarity in rock outcrop ecosystems of the Gulf Islands, British Columbia. Botany 88: 429-434

SAFFORD, H. D., J. H. VIERS \& S. P. HARRISON.2005.
Serpentine endemism in the California flora: a database of serpentine affinity. Madroño 52: 222257.

SCARANO, F. 2007. Rock outcrop vegetation in Brazil: a brief overview. Revista Brasil. Bot. 30: 561-568.

SCHENK, H.J. \& R.B. JACKSON. 2002. Rooting depths, lateral spreads, and below-ground/above-ground allometries of plants in water-limited ecosystems. $J$. Ecol. 90: 480-494.

SEINE, R., S. POREMBSKI \& U. BECKER. 2000. Phytogeography. In: S. POREMBSKI \& W. BARTHLOTT (eds.), Inselbergs, pp. 435-449. Ecological Studies v.146. Springer-Verlag, Berlin.

SELVI, F. 2007. Diversity, geographic variation and conservation of the serpentine flora of Tuscany (Italy). Biodivers. Conserv. 16: 1423-1439.

SFRAGULLA, J. A., D. G. JEREZ \& A. BONALUMI. 1999. Mármoles y otras rocas carbonáticas de Córdoba. En: E. O. ZAPPETTINI (ed.), Recursos Minerales de la República Argentina, pp. 271-295. Anales Instituto de Geología y Recursos Minerales SEGEMAR, Buenos Aires.

SKLENAR, P. \& P. M. JØRGENSEN. 1999. Distribution patterns of páramo plants in Ecuador. J. Biogeogr. 26: 681-691.

SILVERTOWN, J., MC CONWAY, K., GOWING, D., DODD, M., FAY, M., JOSEPH, J.A. \& K. DOLPHIN. 2006. Absence of phylogenetic signal in the niche structure of meadow plant communities. Proc. R. Soc. Biol. Sc. 273 (1582): 39-44.

SMITH, J. M. B. \& A. M. CLEEF. 1988. Composition and origins of the world's tropicalpine floras. $J$. Biogeogr. 15: 631-645.

SMN, 2014. Servicio Meteorológico Nacional. Mapas Normales Anuales. Temperaturas máximas y mínimas absolutas (1961-1990). http://www.smn. gov.ar $/$ mod $=$ clima\&id $=65 \&$ clave $=$ Anuales

SPEZIALE, K.L. \& C. EZCURRA. 2010. The role of outcrops in the diversity of Patagonian vegetation: relicts of glacial paleofloras? Flora 207: 141-149.

STEVANOVIC, V., K. TAN \& G. LATROU. 2003. Distribution of the endemic Balkan flora on serpentine I. Obligate serpentine endemics. Plant Syst. Evol. 242: 149-170.

STOHLGREN, T. J., D. T. BARNETT \& C. S. CROSIER. 2006. Beyond NAWMA-The North American Weed Management Association Standards. http://www. niiss.org/cwis438/websites/niiss/FieldMethods/ BeyondNAWMA.php

TAMME, R., I. HIIESALU, L. LAANISTO, R. SZAVAKOVATS\& M. PÄRTEL. 2010. Environmental heterogeneity, species diversity and co-existence at different spatial scales. J. Veg. Sci. 21: 796-801.

TER BRAAK, C. J. F. 1995. Ordination. In: R. H. J. JONGMAN, C. J. F. TER BRAAK \& O. F. R. VAN 


\section{J. J. Cantero et al. - Vegetación de afloramientos carbonáticos del centro de Argentina}

TONGEREN (eds.), Data Analysis in Community and Landscape Ecology, pp. 91-173. Cambridge University Press, Cambridge.

TER BRAAK, C. J. F. 2002. Program CANOCO Version 4.5. Biometris - quantitative methods in the life and earth sciences Plant Research International, Wageningen University and Research Centre Box 100, 6700 AC Wageningen.

TSIRIPIDIS, I., A. PAPAIOANNOU, V. SAPOUNIDIS \& E. BERGMEIER. 2010. Approaching the serpentine factor at a local scale - a study in an ultramafic area in northern Greece. Plant Soil 329: 35-50.

WARDLE, J. 1971. The forests and shrublands of the Seaward Kaikoura Range. New Zealand J. Bot. 9: 269-292.

WARDLE, P. 1991. Vegetation of New Zealand. Cambridge University Press, Cambridge.

WILSON, S. D. 2000. Heterogeneity, diversity, and scale in plant communitie. In: M. J. HUTCHINGS, E. A. JOHN \& A. J. STEWART (eds.), The ecological consequences of habitat heterogeneity, pp. 53-69. Blackwell, Oxford.

WISER, S. K. \& R. P. BUXTON. 2009. Montane outcrop vegetation of Banks Peninsula, South Island, New Zealand. New Zealand J. Ecol. 33: 164-176.

WISER, S. K. \& R. P. BUXTON. 2008. Context matters: Matrix vegetation influences native and exotic species composition on habitat islands. Ecology 89: 380-391.

WISER, S. K., R. K. PEET \& P. S. WHITE. 1996. Highelevation rock outcrop vegetation of the Southern Appalachian Mountains. J. Veget. Sci. 7: 703-722.
ZAK, M. \& M. CABIDO. 2004. Do subtropical seasonal forests in the Gran Chaco, Argentina, have a future? Biolog. Conserv. 120: 589-598.

ZOBEL, M. 1997. The relative role of species pools in determining plant species richness: an alternative explanation of species coexistence? Trends Ecol. Evol. 12: 266-269.

ZULOAGA, F. O. \& O. MORRONE 1999a. Catálogo de las Plantas Vasculares de la República Argentina. I. Monogr. Syst. Bot. Missouri Bot. Gard. 60: 1-323.

ZULOAGA, F. O. \& O. MORRONE 1999b. Catálogo de las Plantas Vasculares de la República Argentina. II. Monogr. Syst. Bot. Missouri Bot. Gard. 74: 1-1269.

ZULOAGA, F. O., E. G. NICORA, Z. RÚGOLO DE AGRASAR, O. MORRONE, J. PENSIERO \& A. M. CIALDELLA. 1994. Catálogo de la Familia Poaceae en la República Argentina. Monogr. Syst. Bot. Missouri Bot. Gard. 47: 1-178.

ZULOAGA, F. O., O. MORRONE \& M. J. BELGRANO. 2008. Catálogo de Las Plantas Vasculares Del Cono Sur: (Argentina, Sur de Brasil, Chile, Paraguay y Uruguay). Monogr. Syst. Bot. Missouri Bot. Gard.107 (I-II-III): 1-3348.

Recibido el 21 de marzo de 2014, aceptado el 17 de junio de 2014. 


\section{ApÉNDICE}

Listado de las especies de plantas vasculares relevadas en el área de estudio y ordenadas sus correspondientes familias; * indica las especies endémicas; Abreviaturas de formas de vida: árboles (A), arbustos (AR), cactáceas globulares (CG), enredaderas (E), hierbas anuales (HA), helechos (HE), hierbas perennes caducifolias (HPC), hierbas perennes siempre-verdes (HPS), graminoides (G) gramíneas en mata (GM); \% Fr. Rel: porcentaje de frecuencia relativa al total de censos realizados.

\begin{tabular}{|c|c|c|c|}
\hline Taxón & Forma de vida & \% Fr. Rel. & Abreviatura \\
\hline \multicolumn{4}{|l|}{ Acanthaceae } \\
\hline Stenandrium diphyllum Nees & HPS & 45,10 & Stendiph \\
\hline Stenandrium dulce (Cav.) Nees & HPS & 0,98 & Stendulc \\
\hline \multicolumn{4}{|l|}{ Amaranthaceae } \\
\hline Alternanthera pumila O. Stützer* & HPS & 13,73 & Altepumi \\
\hline \multicolumn{4}{|l|}{ Amaryllidaceae } \\
\hline Zephyranthes filifoliaHerb. ex Kraenzl.* & HPC & 4,90 & Zephfili \\
\hline \multicolumn{4}{|l|}{ Anacardiaceae } \\
\hline Schinus fasciculatus (Griseb.) I.M. Johnst. var. fasciculatus & A & 1,47 & Schifasc \\
\hline \multicolumn{4}{|l|}{ Apiaceae } \\
\hline Eryngium nudicaule Lam. & HPS & 0,49 & Erynnudi \\
\hline \multicolumn{4}{|l|}{ Apocynaceae } \\
\hline Asclepias mellodora A. St.-Hil. & HPC & 1,47 & Asclmell \\
\hline Mandevilla petraea (A. St.-Hil.) Pichon & $\mathrm{E}$ & 1,96 & Mandpetr \\
\hline \multicolumn{4}{|l|}{ Aspleniaceae } \\
\hline Asplenium gilliesii Hook. & $\mathrm{HE}$ & 1,47 & Asplgill \\
\hline Asplenium resiliens Kunze & HE & 0,49 & Asplresi \\
\hline \multicolumn{4}{|l|}{ Asteraceae } \\
\hline Acanthostyles buniifolius (Hook. \&Arn.) R.M. King \& H. Rob. & AR & 2,45 & Acanbuni \\
\hline Acmella decumbens (Sm.) R.K. Jansen var. decumbens & HPS & 6,86 & Acmedecu \\
\hline Baccharis aliena (Spreng.) Joch. Müller & AR & 0,98 & Hetealie \\
\hline Baccharis cordobensis Heering* & AR & 3,92 & Bacsess \\
\hline Baccharis flabellata Hook. \& Arn*. & AR & 1,47 & Bacflabe \\
\hline Chaptalia integerrima (Vell.) Burkart & HPS & 0,49 & Chapinte \\
\hline Flourensia oolepis S.F. Blake* & AR & 3,43 & Flouoole \\
\hline Gamochaeta falcata (Lam.) Cabrera & HPC & 2,45 & Gamofalc \\
\hline Gamochaeta pensylvanica (Willd.) Cabrera & HA & 0,49 & Gamoapen \\
\hline $\begin{array}{l}\text { Grindelia pulchella Dunal var. discoidea (Hook. \&Arn.) A. } \\
\text { Bartoli \& Tortosa }\end{array}$ & HPS & 0,98 & Grinpulc \\
\hline Gutierrezia gilliesii Griseb.* & AR & 31,37 & Gutigill \\
\hline Hyaloseris cinerea (Griseb.) Griseb. var. tomentella Griseb. ${ }^{*}$ & AR & 0,98 & Hyalcine \\
\hline Hypochaeris caespitosa Cabrera* & HPS & 0,49 & Hypocaes \\
\hline Lucilia acutifolia (Poir.) Cass. & HPS & 0,49 & Luciacut \\
\hline
\end{tabular}


J. J. Cantero et al. - Vegetación de afloramientos carbonáticos del centro de Argentina

\begin{tabular}{|c|c|c|c|}
\hline Taxón & Forma de vida & $\%$ Fr. Rel. & Abreviatura \\
\hline Noticastrum marginatum (Kunth) Cuatrec. & HPS & 20,59 & Notimarg \\
\hline Parthenium hysterophorus L. & HPC & 3,43 & Parthyst \\
\hline Porophyllum obscurum (Spreng.) DC. & HPS & 26,96 & Poroobsc \\
\hline Pseudognaphalium gaudichaudianum (DC.) Anderb. & $\mathrm{HA}$ & 3,43 & Pseugaud \\
\hline Stevia satureiifolia (Lam.) Sch. Bip. var. satureiifolia & HPS & 87,25 & Stevsatu \\
\hline Thelesperma megapotamicum (Spreng.) Kuntze & HPS & 29,41 & Thelmega \\
\hline Thymophylla pentachaeta (DC.) Small* & HPC & 13,73 & Thympent \\
\hline Trichocline plicata Hook. \& Arn.* & HPS & 35,29 & Tricplic \\
\hline Trichocline reptans (Wedd.) Hieron. & HPS & 1,96 & Tricrept \\
\hline Trichocline sinuata (D. Don) Cabrera* & HPS & 0,49 & Tricsinu \\
\hline $\begin{array}{l}\text { Trixis divaricata (Kunth) Spreng. ssp. discolor (D. Don) } \\
\text { Katinas }\end{array}$ & HPS & 0,49 & Trixdiva \\
\hline Vernonanthura nudiflora (Less.) H. Rob. f. nudiflora & HPS & 1,47 & Vernnudi \\
\hline Zexmenia buphtalmiflora (Lorentz) Ariza* & HPS & 14,22 & Zexmbuph \\
\hline \multicolumn{4}{|l|}{ Boraginaceae } \\
\hline Heliotropium campestre Griseb. & HPC & 2,45 & Helicamp \\
\hline Phacelia pinnatifida Griseb. ex Wedd. & HPC & 26,96 & Phacepin \\
\hline \multicolumn{4}{|l|}{ Brassicaceae } \\
\hline Lepidium bonariense L. & $\mathrm{HA}$ & 1,96 & Lepibona \\
\hline Physaria mendocina (Phil.) O'Kane \& Al-Shehbaz & HPC & 66,18 & Physmend \\
\hline \multicolumn{4}{|l|}{ Buddlejaceae } \\
\hline Buddleja cordobensis Griseb.* & $A R$ & 0,49 & Buddcord \\
\hline \multicolumn{4}{|l|}{ Cactaceae } \\
\hline $\begin{array}{l}\text { Gymnocalycium monvillei (Lem.) Britton \& Rose ssp. } \\
\text { monvillei* }\end{array}$ & CG & 0,49 & Gymmonho \\
\hline Gymnocalycium mostii (Gürke) Britton \& Rose & CG & 0,49 & Gymmonmo \\
\hline \multicolumn{4}{|l|}{ Calceolariaceae } \\
\hline Calceolaria parviflora Benth. & HPC & 0,49 & Calcparv \\
\hline \multicolumn{4}{|l|}{ Caryophyllaceae } \\
\hline Silene argentina (Pax) Bocquet* & HPC & 1,96 & Sileaarg \\
\hline Wahlenbergia linarioides (Lam.) A. DC. & HPC & 0,98 & Wahllina \\
\hline \multicolumn{4}{|l|}{ Commelinaceae } \\
\hline Commelina erecta L. var. angustifolia (Michx.) Fernald & HPS & 0,49 & Commerec \\
\hline \multicolumn{4}{|l|}{ Convolvulaceae } \\
\hline Bonamia sericea (Griseb.) Hallier f. var. sericea & HPS & 3,43 & Bonaseri \\
\hline Dichondra sericea Sw. var. sericea & HPS & 15,20 & Dichseri \\
\hline Evolvulus sericeus Sw. var. sericeus & HPS & 34,80 & Evolseri \\
\hline \multicolumn{4}{|l|}{ Cyperaceae } \\
\hline Bulbostylis juncoides (Vahl) Kük. ex Herter var. juncoides & G & 0,98 & Bulbjunc \\
\hline
\end{tabular}


Bol. Soc. Argent. Bot. 49 (4) 2014

\section{Ephedraceae}

Ephedra triandra Tul. emend. J.H. Hunz.

$$
\text { AR }
$$

0,98

Ephetria

\section{Euphorbiaceae}

Croton argentinus Müll. Arg.

$\begin{array}{ccc}\text { HPS } & 12,75 & \begin{array}{c}\text { Crotarge } \\ \text { HPS }\end{array} \\ \text { A } & 2,45 & \begin{array}{c}\text { Euphahie } \\ \text { Sebacomm }\end{array} \\ \text { G } & 1,47 & \\ \text { G } & 5,88 & \text { Sisyamic } \\ & 80,88 & \text { Sisyungu }\end{array}$

Euphorbia hieronymi Subils*

Sebastiania commersoniana (Baill.) L.B. Sm. \& Downs

\section{Iridaceae}

Sisyrinchium unguiculatum Griseb. *

Sisyrinchium micranthum Cav. ssp. valdivianum (Phil.)

Sisyungu

Ravenna

\section{Lamiaceae}

Hedeoma multiflorum Benth.

HPS

\section{Leguminosae}

Acacia caven (Molina) Molina var. caven

Acacia praecox Griseb.

Adesmia cordobensis Burkart var. cordobensis*

Adesmia incana Vogel var. grisea (Hook. f.) Burkart

Aeschynomene histrix Poir. var. histrix

Apurimacia dolichocarpa (Griseb.) Burkart*

Astragalus distinens Macloskie

Astragalus parodii I.M. Johnst.*

Dalea elegans Gillies ex Hook. \& Arn. var. elegans*

Galactia glaucophylla Harms*

Prosopis torquata (Cav. ex Lag.) DC.*

Rhynchosia senna Gillies ex Hook. var. senna

\section{Linaceae}

Linum scoparium Griseb.

\section{Malvaceae}

Ayenia cordobensis (Hieron.) Hieron. ${ }^{*}$

Krapovickasia flavescens (Cav.) Fryxell

Sphaeralcea cordobensis Krapov. *

\section{Nyctaginaceae}

Allionia incarnata L.

Boerhavia diffusa L. var. diffusa

\section{Oleaceae}

Menodora integrifolia (Cham. \& Schltdl.) Steud. var. integrifolia

\section{Orobanchaceae}

Agalinis genistifolia (Cham. \& Schltdl.) D'Arcy
HPS

A

A

HPS

HPS

HPS

AR

HPC

HPC

HPS

E

A

E

$\begin{array}{ccc}\text { HPS } & 9,80 & \text { Ayencord } \\ \text { HPS } & 15,20 & \text { Krapflav } \\ \text { HPC } & 0,98 & \text { Sphacord }\end{array}$

Acacaven

Acaprae

Adescord

Adesinca

Aeschist

Apurdoli

1,47

Astrdist

10,29

Astrparo

1,96 Daleeleg

2,94

Galaglau

3,92

Prostorq

17,16

Rhysenna

27,45 Linuscop

HPC

9,80

Alliinca

HPC

5,39

Boerdiff

HPS

0,49

Menointe

9,80

Agalgeni 
J. J. Cantero et al. - Vegetación de afloramientos carbonáticos del centro de Argentina

\begin{tabular}{|c|c|c|c|}
\hline Taxón & Forma de vida & $\%$ Fr. Rel. & Abreviatura \\
\hline \multicolumn{4}{|l|}{ Passifloraceae } \\
\hline Passiflora foetida L. var. foetida & E & 0,49 & Passfoet \\
\hline Turnera sidoides L. ssp. pinnatifida (Juss. ex Poir.) Arbo & HPS & 23,04 & Turnsido \\
\hline \multicolumn{4}{|l|}{ Plantaginaceae } \\
\hline Plantago argentina Pilg. & HPS & 9,80 & Planarge \\
\hline Plantago brasiliensis Sims* & HPS & 18,14 & Planbras \\
\hline \multicolumn{4}{|l|}{ Poaceae } \\
\hline Aristida spegazzinii Arechav. & G & 36,76 & Arisspeg \\
\hline Bothriochloa springfieldii (Gould) Parodi & GM & 2,94 & Bothspri \\
\hline $\begin{array}{l}\text { Bouteloua curtipendula (Michx.) Torr. var. caespitosa Gould } \\
\text { \& Kapadia }\end{array}$ & G & 6,37 & Boutcurt \\
\hline Bouteloua megapotamica (Spreng.) Kuntze & G & 5,39 & Boutmega \\
\hline Bromus auleticus Trin. ex Nees & G & 3,92 & Bromaule \\
\hline Chascolytrum paleapiliferum (Parodi) Matthei & G & 7,35 & Chaspale \\
\hline Chloris ciliata Sw. f. ciliata & G & 1,47 & Chlocili \\
\hline Danthonia cirrata Hack. \& Arechav. & G & 8,82 & Dantcirr \\
\hline Elionurus muticus (Spreng.) Kuntze & G & 0,49 & Eliomuti \\
\hline Eragrostis lugens Nees & G & 41,67 & Eragluge \\
\hline $\begin{array}{l}\text { Erioneuron pilosum (Buckley) Nash var. longearistatum } \\
\text { (Kurtz) Anton* }\end{array}$ & G & 18,14 & Eriopilo \\
\hline Jarava juncoides (Speg.) Peñailillo* & G & 57,84 & Jarajunc \\
\hline Koeleria kurtzii Hack. ex Kurtz emend. A.M. Molina & G & 4,41 & Koelkurt \\
\hline Melica eremophila Torres & G & 0,49 & Melierem \\
\hline Nassella filiculmis (Delile) Barkworth & GM & 1,96 & Nassfili \\
\hline Nassella hunzikeri (Caro) Barkworth* & G & 6,37 & Nasshunz \\
\hline Nassella sanluisensis (Speg.) Barkworth* & GM & 7,84 & Nasssanl \\
\hline Nassella stuckertii (Hack.) Barkworth* & G & 15,69 & Nassstuc \\
\hline Pappophorum phillippianum Parodi & GM & 1,96 & Pappphil \\
\hline Paspalum humboldtianum Flüggé & G & 8,33 & Pasphumb \\
\hline Piptochaetium napostaense (Speg.) Hack. ${ }^{*}$ & G & 3,43 & Piptnapo \\
\hline Poa lanuginosa Poir. var. lanuginosa & G & 0,98 & Poalanu \\
\hline Schizachyrium salzmannii (Trin. ex Steud.) Nash & G & 0,49 & Schisalz \\
\hline Schizachyrium sanguineum (Retz.) Alston & G & 65,69 & Schiasan \\
\hline Schizachyrium spicatum (Spreng.) Herter & G & 11,76 & Schispic \\
\hline Sorghastrum pellitum (Hack.) Parodi & G & 6,37 & Sorgpell \\
\hline \multicolumn{4}{|l|}{ Polygalaceae } \\
\hline Monnina dictyocarpa Griseb. & HPS & 53,92 & Mondicty \\
\hline Polygala stenophylla A. Gray* & HPS & 10,29 & Polysten \\
\hline
\end{tabular}


Bol. Soc. Argent. Bot. 49 (4) 2014

\begin{tabular}{|c|c|c|c|}
\hline Taxón & Forma de vida & $\%$ Fr. Rel. & Abreviatura \\
\hline Polygala subandina Phil. & HPS & 29,41 & Polysuba \\
\hline \multicolumn{4}{|l|}{ Portulacaceae } \\
\hline Portulaca eruca Hauman & HPC & 3,43 & Poreruca \\
\hline \multicolumn{4}{|l|}{ Pteridaceae } \\
\hline Argyrochosma nivea (Poir.) Windham var. nivea & $\mathrm{HE}$ & 0,49 & Argynive \\
\hline \multicolumn{4}{|l|}{ Rhamnaceae } \\
\hline Colletia spinosissima J.F. Gmel. & AR & 2,45 & Collspin \\
\hline \multicolumn{4}{|l|}{ Rosaceae } \\
\hline Margyricarpus pinnatus (Lam.) Kuntze & AR & 7,35 & Margpinn \\
\hline \multicolumn{4}{|l|}{ Rubiaceae } \\
\hline $\begin{array}{l}\text { Borreria eryngioides Cham. \& Schltdl. var. ostenii (Standl.) } \\
\text { E.L. Cabral \& Bacigalupo* }\end{array}$ & HPS & 10,78 & Borreryn \\
\hline $\begin{array}{l}\text { Galium richardianum (Gillies ex Hook. \&Arn.) Endl. ex Walp. } \\
\text { ssp. richardianum }\end{array}$ & $\mathrm{E}$ & 48,53 & Galirich \\
\hline Mitracarpus megapotamicus (Spreng.) Kuntze & HPS & 28,92 & Mitrameg \\
\hline \multicolumn{4}{|l|}{ Selaginellaceae } \\
\hline Selaginella peruviana (Milde) Hieron. & HE & 4,41 & Selaperu \\
\hline Selaginella sellowii Hieron. & $\mathrm{HE}$ & 11,27 & Selasell \\
\hline \multicolumn{4}{|l|}{ Solanaceae } \\
\hline Nierembergia aristata D. Don & HPC & 2,45 & Nieraris \\
\hline Physalis viscosa $\mathrm{L}$. & HPC & 0,98 & Physvisc \\
\hline Solanum stuckertii Bitter & AR & 0,49 & Solastuc \\
\hline \multicolumn{4}{|l|}{ Verbenaceae } \\
\hline Glandularia platensis (Spreng.) Schnack \& Covas & HPS & 0,49 & Glanplat \\
\hline Glandularia pulchella (Sweet) Tronc. var. pulchella & HPS & 22,06 & Glanpulc \\
\hline Glandularia subincana Tronc. & HPS & 13,73 & Glanasub \\
\hline $\begin{array}{l}\text { Junellia bisulcata (Hayek) Moldenke var. campestris (Griseb.) } \\
\text { Botta* }^{*}\end{array}$ & AR & 0,49 & Junebisu \\
\hline Lantana grisebachii Stuck. ex Seckt var. grisebachii & AR & 0,49 & Lantgris \\
\hline Lippia junelliana (Moldenke) Tronc. & AR & 0,49 & Lippjune \\
\hline \multicolumn{4}{|l|}{ Violaceae } \\
\hline Hybanthus serratus (Phil.) Hassl. ${ }^{*}$ & HPS & 1,47 & Hybaserr \\
\hline
\end{tabular}

